\title{
Mitigation of Bullwhip Effect in Closed-Loop Supply Chain Based on Fuzzy Robust Control Approach
}

\author{
Songtao Zhang $\mathbb{D D}^{1}$ and Min Zhang $\mathbb{D}^{2}$ \\ ${ }^{1}$ School of Logistics, Linyi University, Linyi 276005, China \\ ${ }^{2}$ Library, Linyi University, Linyi 276005, China \\ Correspondence should be addressed to Min Zhang; zm0205@163.com
}

Received 25 July 2019; Revised 23 November 2019; Accepted 16 December 2019; Published 21 January 2020

Guest Editor: Raúl Villafuerte-Segura

Copyright (c) 2020 Songtao Zhang and Min Zhang. This is an open access article distributed under the Creative Commons Attribution License, which permits unrestricted use, distribution, and reproduction in any medium, provided the original work is properly cited.

\begin{abstract}
Uncertainties and lead times make the closed-loop supply chain (CLSC) more complex, less stable, and then the bullwhip effect (BE) will become more intense. This paper will address a fuzzy robust control (FRC) approach to mitigate the BE in the uncertain CLSC with lead times. For the reverse channels for products in the CLSC, the customers' used products are recycled by both the manufacturer and the third party recovery provider, and new products bought by customers within a certain period of time can be returned to the retailer. In the CLSC system, the state transformation equations of the inventories and the total operation cost are set up. A new FRC approach is proposed to mitigate the BE and realize the robust stability of the uncertain CLSC with lead times. A simulation example verifies the mitigation effect of the BE under the proposed FRC approach.
\end{abstract}

\section{Introduction}

Whether governments construct green supply chains through governmental interventions [1] or enterprises undertake social responsibility through social work donation [2], the environment can be more friendly. Therefore, both governments and enterprises have been paying more and more attention to the closed-loop supply chain (CLSC) which can achieve sustainable development [3].

The complexity of the business environment results in many uncertainties in the CLSC. Especially in the reverse supply chain, there are uncertainties in quality, quantity, and time of the recycled products, which lead to the uncertainty of remanufacturing profit. For example, ReCellular, one of the largest phone remanufacturers in the United States, divides used phones into six quality levels for remanufacturing. Uncertainty is one of the main reasons for the existence of bullwhip effect (BE), and the other reason is lead time. If $\mathrm{BE}$ cannot be effectively mitigated, the operating efficiency of the CLSC will be reduced, and then the operating cost will be increased. In serious cases, the CLSC will collapse. Therefore, for the mitigation issue of the BE in the CLSC with uncertainties and lead times, we will propose a fuzzy robust control (FRC) approach to reduce the $\mathrm{BE}$ and realize the robust stability of the CLSC.

The remainder of this paper is formulated as follows: Section 2 offers a review of the related literature. Section 3 puts forward a kind of Takagi-Sugeno fuzzy model for the CLSC with uncertainties and lead times. Section 4 addresses a FRC approach to mitigate the BE in the CLSC system. The simulation studies are carried out in Section 5. The conclusions and future research directions are given in Section 6.

\section{Literature Review}

In the CLSC, used products can be recycled by various subjects. For example, the used products can be recycled by the manufacturer [4] or by the retailer [5]. Wei and Zhao [6] studied the decision-making issue of the used products recycled by the manufacturer, the retailer, or the third party recovery provider (3PRP). Furthermore, recycling used products can be performed by multiple subjects at the same time to realize more convenient for customers and more efficient for enterprises. This recycling pattern is called hybrid recycling. Therefore, some scholars have 
been devoting themselves to the study of the CLSC with hybrid recycling channels; for example, based on hybrid recycling channels, Allah et al. [7] investigated the pricing strategies of the CLSCs with the single-channel forward supply chain and with the dual-channel forward supply chain; Shi and Ma [8] recycled the used medical equipment through hybrid recycling models; under hybrid recycling channels, Ma and Liu [9] analyzed the optimal profit of the CLSC.

For the studies mentioned above, [7-9] did not consider the impacts of uncertainties and lead times on the CLSC system with hybrid recycling channels. However, uncertainties and lead times are two important factors leading to BE. Therefore, many scholars studied the BE caused by uncertainties or lead times; for example, by simulationbased approach, Do et al. [10] quantified the BE with different demands and stochastic lead times; for a two-echelon serial supply chain, Agrawal et al. [11] compared the effects of lead time reduction and information sharing on the mitigation of the BE; using the statistical method, Kim et al. [12] considered the stochastic lead time and provided expressions for quantifying the BE both with information sharing and without information sharing; Modak and Kelle [13] used a hybrid all-unit quantity discount along a franchise fee contract to mitigate the $\mathrm{BE}$ in the dual-channel supply chain with the delivery time and stochastic demand; $\mathrm{Li}$ and Liu [14] explored the mitigation of the BE in the supply chain with uncertainties and the vendor order placement lead time.

In recent years, approaches based on control theory have been widely applied to mitigate the $\mathrm{BE}$, such as Model Predictive Control (MPC) approach [15], Internal Model Control (IMC) approach [16], Proportional plus Integral Control (PIC) approach [17], and Common Robust Control (CRC) approach [18]. Among these mitigation approaches, the MPC approach, the IMC approach, and the PIC approach cannot implement the switching control, and the CRC approach can only perform the conventional switching control but cannot achieve the flexible switching control of the FRC approach.

Up to now, research on the mitigation of the $\mathrm{BE}$ in the CLSC with uncertainties and lead times has not yet been found. But, a fuzzy control approach has been applied to mitigate the BE in uncertain CLSC with hybrid recycling channels by Zhang et al. [19]. Therefore, we will follow the research ideas in [19] to address a control approach for mitigating the $\mathrm{BE}$ of the CLSC with uncertainties and lead times. The critical contributions of this paper comparing to [19] are listed as follows.

2.1. Lead Times Are Included in the CLSC Models. There are the manufacturer's production lead time and recycling lead time in the manufacturer' inventory model; the retailer's ordering lead time and the 3PRP's recycling lead time are, respectively, considered in the retailer's inventory model and in the 3PRP's inventory model; all lead times are included in the total operation cost model of the CLSC.

2.2. An Additional Takagi-Sugeno Fuzzy Controller for Lead Times Is Designed. We will design an additional TakagiSugeno fuzzy negative feedback controller, which is the product of the inventory feedback gains with lead times and the inventory variables with lead times.

2.3. A New Fuzzy Control Approach Is Put Forward. We will put forward a new FRC approach which can effectively mitigate the $\mathrm{BE}$ caused by uncertainties and lead times and ensure the stability of the CLSC system. LMIs (linear matrix inequalities) to be solved in this paper are more complex than those in [19].

\section{CLSC Model}

3.1. CLSC Model with Uncertainties and Lead Times. As shown in Figure 1, this paper constructs a CLSC model that includes a manufacturer, a retailer, a 3PRP, and customers. Because products recycled by both the manufacturer and the 3PRP are the most effective pattern among hybrid recycling channels [20], for reverse supply chain in our model, the manufacturer and the 3PRP simultaneously recycle customers' used products, and the retailer allows customers to return new products within a certain period of time.

Based on Figure 1, considering the uncertain system parameters and lead times, we set up the inventory equations of the CLSC as follows:

$$
\left\{\begin{array}{l}
x_{1}(k+1)=x_{1}(k)+u_{1}(k)+u_{1}\left(k-\tau_{1}\right)+u_{3}(k)+u_{3}\left(k-\tau_{3}\right)+(\eta+\Delta \eta) x_{4}(k)-u_{2}(k), \\
x_{2}(k+1)=x_{2}(k)+u_{2}(k)+u_{2}\left(k-\tau_{2}\right)+(\mu+\Delta \mu) x_{3}(k)-w_{1}(k), \\
x_{3}(k+1)=x_{3}(k)+w_{1}(k)-u_{3}(k)-u_{4}(k)-(\mu+\Delta \mu) x_{3}(k), \\
x_{4}(k+1)=x_{4}(k)+u_{4}(k)+u_{4}\left(k-\tau_{4}\right)-(\eta+\Delta \eta) x_{4}(k)-(\lambda+\Delta \lambda) x_{4}(k) .
\end{array}\right.
$$




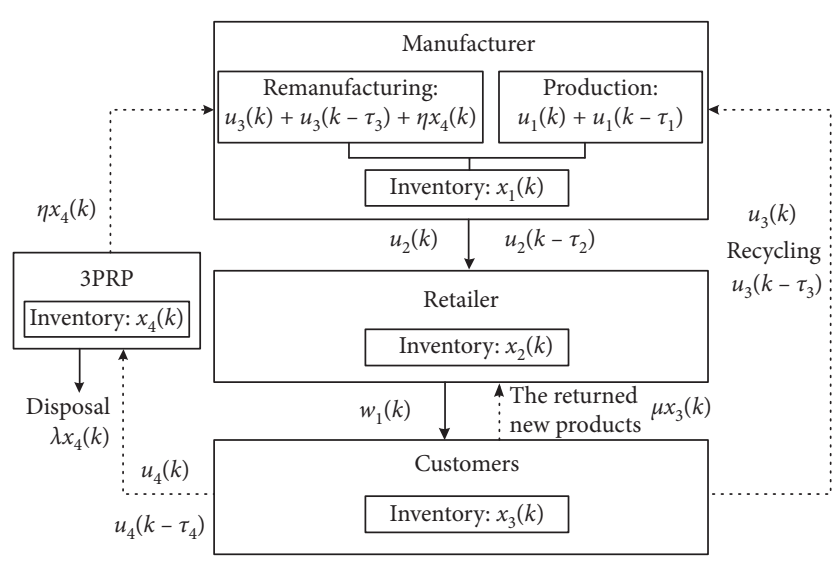

Figure 1: CLSC model.

Accordingly, we set up the total cost equation of the CLSC as follows:

$$
\begin{aligned}
z(k)= & \left(c_{h 1}+\Delta c_{h 1}\right) x_{1}(k)+\left(c_{h 2}+\Delta c_{h 2}\right) x_{2}(k)+\left(c_{h 3}+\Delta c_{h 3}\right) x_{4}(k) \\
& +\left(c_{n}+\Delta c_{n}\right)\left[u_{1}(k)+u_{1}\left(k-\tau_{1}\right)\right] \\
& +\left(c_{r}+\Delta c_{r}\right)\left[u_{3}(k)+u_{3}\left(k-\tau_{3}\right)+(\eta+\Delta \eta) x_{4}(k)\right] \\
& +\left(c_{m}+\Delta c_{m}\right)\left[u_{3}(k)+u_{3}\left(k-\tau_{3}\right)\right] \\
& +\left(c_{t}+\Delta c_{t}\right)\left[u_{4}(k)+u_{4}\left(k-\tau_{4}\right)\right] \\
& +\left(c_{m t}+\Delta c_{m t}\right)(\eta+\Delta \eta) x_{4}(k) \\
& +\left(c_{q}+\Delta c_{q}\right)(\mu+\Delta \mu) x_{3}(k) \\
& +\left(c_{d}+\Delta c_{d}\right)(\lambda+\Delta \lambda) x_{4}(k) \\
& +\left(c_{s}+\Delta c_{s}\right)\left[u_{2}(k)+u_{2}\left(k-\tau_{2}\right)\right] .
\end{aligned}
$$

Equations (1) and (2) are described by the deviation values (deviation value $=$ actual value - nominal value).

In addition, based on different inventory statuses, each node enterprise will design corresponding production patterns or ordering patterns, which leads to some different models in different periods. Then, the $i$ th model of the CLSC can be shown as follows:

$$
\left\{\begin{array}{l}
\mathbf{x}(k+1)=\left(\mathbf{A}_{i}+\Delta \mathbf{A}_{i}\right) \mathbf{x}(k)+\left(\mathbf{B}_{i}+\Delta \mathbf{B}_{i}\right) \mathbf{u}(k)+\sum_{e=1}^{4}\left(\mathbf{B}_{i e}+\Delta \mathbf{B}_{i e}\right) \mathbf{u}\left(k-\tau_{e}\right)+\left(\mathbf{B}_{w i}+\Delta \mathbf{B}_{w i}\right) \mathbf{w}(k), \\
z(k)=\left(\mathbf{C}_{i}+\Delta \mathbf{C}_{i}\right) \mathbf{x}(k)+\left(\mathbf{D}_{i}+\Delta \mathbf{D}_{i}\right) \mathbf{u}(k)+\sum_{e=1}^{4}\left(\mathbf{D}_{i e}+\Delta \mathbf{D}_{i e}\right) \mathbf{u}\left(k-\tau_{e}\right)
\end{array}\right.
$$

where $\mathbf{x}^{\mathrm{T}}(k)=\left[x_{1}(k), x_{2}(k), x_{3}(k), x_{4}(k)\right], \mathbf{u}^{\mathrm{T}}(k)=\left[u_{1}(k)\right.$, $\left.u_{2}(k), u_{3}(k), u_{4}(k)\right], \mathbf{u}^{\mathrm{T}}\left(k-\tau_{e}\right)=\left[u_{1}\left(k-\tau_{1}\right), u_{2}\left(k-\tau_{2}\right)\right.$, $\left.u_{3}\left(k-\tau_{3}\right), u_{4}\left(k-\tau_{4}\right)\right]$, and $\mathbf{w}^{\mathrm{T}}(k)=\left[w_{1}(k), 0,0,0\right] ; \mathbf{A}_{i}$ denotes the inventory status coefficient matrix; $\mathbf{B}_{i}$ denotes the production, ordering, and recycling coefficient matrix; $\mathbf{B}_{i e}$ denotes the production, ordering, and recycling coefficient matrix with lead times; $\mathbf{B}_{w i}$ denotes the coefficient matrix of the customers' demand; $\mathbf{C}_{i}$ denotes the coefficient matrix of the inventory cost, the cost of the new products returned, the disposal cost, part of remanufacturing cost, and part of recycling cost; $\mathbf{D}_{i}$ denotes the coefficient matrix of production cost, ordering cost, part of remanufacturing cost, and part of recycling cost; $\mathbf{D}_{i e}$ denotes the coefficient matrix of production cost, ordering cost, part of remanufacturing cost, and part of recycling cost with lead times; and $\Delta \mathbf{A}_{i}, \Delta \mathbf{B}_{i}, \Delta \mathbf{B}_{i e}, \Delta \mathbf{B}_{w i}, \Delta \mathbf{C}_{i}, \Delta \mathbf{D}_{i}$, and $\Delta \mathbf{D}_{i e}$, respectively, denote the corresponding uncertain matrices. 
3.2. Takagi-Sugeno Fuzzy Model of CLSC. In the process of wide variation of the inventory level, switching activities will take place among models to maintain a reasonable inventory level in each node enterprise. In order to effectively mitigate the BE of the CLSC, we will utilize Takagi-Sugeno fuzzy systems [21] to implement the flexible switching in the CLSC. Then, Model (3) is transformed into the following discrete Takagi-Sugeno fuzzy model: then

$$
\left\{\begin{array}{l}
\mathbf{x}(k+1)=\left(\mathbf{A}_{i}+\Delta \mathbf{A}_{i}\right) \mathbf{x}(k)+\left(\mathbf{B}_{i}+\Delta \mathbf{B}_{i}\right) \mathbf{u}(k)+\sum_{e=1}^{g}\left(\mathbf{B}_{i e}+\Delta \mathbf{B}_{i e}\right) \mathbf{u}\left(k-\tau_{e}\right)+\left(\mathbf{B}_{w i}+\Delta \mathbf{B}_{w i}\right) \mathbf{w}(k), \\
z(k)=\left(\mathbf{C}_{i}+\Delta \mathbf{C}_{i}\right) \mathbf{x}(k)+\left(\mathbf{D}_{i}+\Delta \mathbf{D}_{i}\right) \mathbf{u}(k)+\sum_{e=1}^{g}\left(\mathbf{D}_{i e}+\Delta \mathbf{D}_{i e}\right) \mathbf{u}\left(k-\tau_{e}\right), \\
\mathbf{x}(k)=\varphi(k), \quad k \in\{0,1, \ldots, N\},
\end{array}\right.
$$

where $\mathbf{w}(k) \in l_{2}[\mathbf{0}, \infty) ; \boldsymbol{\varphi}(k)$ denotes the initial condition of the CLSC; $\quad \mathbf{x}^{\mathrm{T}}(k)=\left[x_{1}(k), x_{2}(k), \ldots, x_{n}(k)\right] ; \quad \mathbf{u}^{\mathrm{T}}(k)=$ $\left[u_{1}(k), u_{2}(k), \ldots, u_{n}(k)\right] ; \quad \mathbf{u}^{\mathrm{T}}\left(k-\tau_{e}\right)=\left[u_{1}\left(k-\tau_{1}\right), u_{2}(k-\right.$ $\left.\left.\tau_{2}\right), \ldots, u_{n}\left(k-\tau_{g}\right)\right] ; \quad$ and $\quad \mathbf{w}^{\mathrm{T}}(k)=\left[w_{1}(k), w_{2}(k), \ldots\right.$, $\left.w_{n}(k)\right]$.

By singleton fuzzifier, product inference, and centreaverage defuzzifer, Model (4) can be expressed as follows:

$$
\left\{\begin{array}{l}
\mathbf{x}(k+1)=\sum_{i=1}^{r} h_{i}(\mathbf{x}(k)) \cdot\left[\left(\mathbf{A}_{i}+\Delta \mathbf{A}_{i}\right) \mathbf{x}(k)+\left(\mathbf{B}_{i}+\Delta \mathbf{B}_{i}\right) \mathbf{u}(k)+\sum_{e=1}^{g}\left(\mathbf{B}_{i e}+\Delta \mathbf{B}_{i e}\right) \mathbf{u}\left(k-\tau_{e}\right)+\left(\mathbf{B}_{w i}+\Delta \mathbf{B}_{w i}\right) \mathbf{w}(k)\right] \\
z(k)=\sum_{i=1}^{r} h_{i}(\mathbf{x}(k)) \cdot\left[\left(\mathbf{C}_{i}+\Delta \mathbf{C}_{i}\right) \mathbf{x}(k)+\left(\mathbf{D}_{i}+\Delta \mathbf{D}_{i}\right) \mathbf{u}(k)+\sum_{e=1}^{g}\left(\mathbf{D}_{i e}+\Delta \mathbf{D}_{i e}\right) \mathbf{u}\left(k-\tau_{e}\right)\right]
\end{array}\right.
$$

where $h_{i}(\mathbf{x}(k))=\mu_{i}(\mathbf{x}(k)) / \sum_{i=1}^{r} \mu_{i}(\mathbf{x}(k))$ and $\mu_{i}(\mathbf{x}(k))=$ $\prod_{j=1}^{n} M_{j}^{i}\left(x_{j}(k)\right)$; since $\mu_{i}(\mathbf{x}(k)) \geq 0$, we have $h_{i}(\mathbf{x}(k)) \geq 0$ and $\sum_{i=1}^{r} h_{i}(\mathbf{x}(k))=1$. In the following expressions, $\mathbf{x}(k)$ will be elided from $h_{i}(\mathbf{x}(k))$ for simplicity.

Because of the existence of the membership degree function in Model (5), the flexible switching can be realized among the models of the CLSC.

\section{Mitigation of BE}

4.1. Measurement of BE. We use the following parameter $\gamma$ to represent the mitigation degree of $\mathrm{BE}$ :

$$
\frac{\| \text { total cost of CLSC } \|_{2}}{\| \text { customers' demand } \|_{2}} \leq \gamma \text {. }
$$

From the inequality above, we know that the mitigation degree of BE depends on the size of the total cost of the CLSC and the size of the customers' demand. The lower $\gamma$ is, the smaller BE is.

4.2. FRC Approach. We introduce known constant matrices $\mathbf{H}_{1 i}, \mathbf{H}_{2 i}, \mathbf{E}_{11 i}, \mathbf{E}_{12 i}, \mathbf{E}_{13 i}, \mathbf{E}_{21 i}, \mathbf{E}_{22 i}, \mathbf{L}_{\mathrm{ei}}$, and $\mathbf{O}_{\mathrm{ei}}$ and timevarying uncertain matrices $\mathbf{F}_{1 i}(k)$ and $\mathbf{F}_{2 i}(k)$ to describe the uncertain parameters in the CLSC; $\mathbf{F}_{1 i}(k)$, and $\mathbf{F}_{2 i}(k)$ are Lebesgue-measurable and satisfy $\mathbf{F}_{1 i}^{\mathrm{T}}(k) \mathbf{F}_{1 i}(k) \leq \mathbf{I}$ and
$\mathbf{F}_{2 i}^{\mathrm{T}}(k) \mathbf{F}_{2 i}(k) \leq \mathbf{I}$. Then, we represent the uncertain parameter matrices in Model (5) as follows:

$$
\begin{aligned}
& {\left[\Delta \mathbf{A}_{i}, \Delta \mathbf{B}_{i}, \Delta \mathbf{B}_{w i}, \Delta \mathbf{B}_{i 1}, \ldots, \Delta \mathbf{B}_{i e}, \ldots, \Delta \mathbf{B}_{i g}\right]} \\
& =\mathbf{H}_{1 i} \mathbf{F}_{1 i}(k)\left[\mathbf{E}_{11 i}, \mathbf{E}_{12 i}, \mathbf{E}_{13 i}, \mathbf{L}_{1 i}, \ldots, \mathbf{L}_{\mathrm{ei}}, \ldots, \mathbf{L}_{\mathrm{gi}}\right] ; \\
& {\left[\Delta \mathbf{C}_{i}, \Delta \mathbf{D}_{i}, \Delta \mathbf{D}_{i 1}, \ldots, \Delta \mathbf{D}_{i e}, \ldots, \Delta \mathbf{D}_{i g}\right]} \\
& =\mathbf{H}_{2 i} \mathbf{F}_{2 i}(k)\left[\mathbf{E}_{21 i}, \mathbf{E}_{22 i}, \mathbf{O}_{1 i}, \ldots, \mathbf{O}_{\mathrm{e} i}, \ldots, \mathbf{O}_{g i}\right] .
\end{aligned}
$$

For the fuzzy CLSC system in Model (5), we design the Takagi-Sugeno fuzzy controller as follows.

Controller rule $K^{i}$ : then

If $x_{1}(k)$ is $M_{1}^{i}, \ldots, x_{j}(k)$ is $M_{j}^{i}, \ldots$, and $x_{n}(k)$ is $M_{n}^{i}$,

$$
\begin{cases}\mathbf{u}(k)=-\mathbf{K}_{i} \mathbf{x}(k), & i=1,2, \ldots, r, \\ \mathbf{u}\left(k-\tau_{e}\right)=-\mathbf{K}_{i e} \mathbf{x}\left(k-\tau_{e}\right), & e=1,2, \ldots, g,\end{cases}
$$

where $\mathbf{K}_{i}$ is the inventory feedback gain matrix and $\mathbf{K}_{i e}$ is the inventory feedback gain matrix with lead times. Furthermore, we can obtain the following overall model of Model (8):

$$
\left\{\begin{array}{l}
\mathbf{u}(k)=-\sum_{i=1}^{r} h_{i} \mathbf{K}_{i} \mathbf{x}(k) \\
\mathbf{u}\left(k-\tau_{e}\right)=-\sum_{i=1}^{r} h_{i} \mathbf{K}_{i e} \mathbf{x}\left(k-\tau_{e}\right)
\end{array}\right.
$$


Therefore, introducing Controller (9) into Model (5), we have

$$
\left\{\begin{array}{l}
\mathbf{x}(k+1)=\sum_{i=1}^{r} \sum_{j=1}^{r} h_{i} h_{j}\left[\left(\overline{\mathbf{A}}_{i}-\overline{\mathbf{B}}_{i} \mathbf{K}_{j}\right) \mathbf{x}(k)-\sum_{e=1}^{g} \overline{\mathbf{B}}_{i e} \mathbf{K}_{j e} \mathbf{x}\left(k-\tau_{e}\right)+\overline{\mathbf{B}}_{w i} \mathbf{w}(k)\right], \\
z(k)=\sum_{i=1}^{r} \sum_{j=1}^{r} h_{i} h_{j}\left[\left(\overline{\mathbf{C}}_{i}-\overline{\mathbf{D}}_{i} \mathbf{K}_{j}\right) \mathbf{x}(k)-\sum_{e=1}^{g} \overline{\mathbf{D}}_{i e} \mathbf{K}_{j e} \mathbf{x}\left(k-\tau_{e}\right)\right] .
\end{array}\right.
$$

where $\quad \overline{\mathbf{A}}_{i}=\mathbf{A}_{i}+\Delta \mathbf{A}_{i}, \quad \overline{\mathbf{B}}_{i}=\mathbf{B}_{i}+\Delta \mathbf{B}_{i}, \quad \overline{\mathbf{B}}_{i e}=\mathbf{B}_{i e}+\Delta \mathbf{B}_{i e}$, $\overline{\mathbf{B}}_{w i}=\mathbf{B}_{w i}+\Delta \mathbf{B}_{w i}, \quad \overline{\mathbf{C}}_{i}=\mathbf{C}_{i}+\Delta \mathbf{C}_{i}, \quad \overline{\mathbf{D}}_{i}=\mathbf{D}_{i}+\Delta \mathbf{D}_{i}, \quad$ and $\overline{\mathbf{D}}_{i \mathrm{e}}=\mathbf{D}_{i e}+\Delta \mathbf{D}_{i e}$.

For the further analysis, we introduce the following Definitions, Property, and Lemma.

Definition 1 (see [22]). A cluster of fuzzy sets $\left\{F_{j}^{m}, m\right.$ $\left.=1,2, \ldots, q_{j}\right\}$ are said to be a standard fuzzy partition in the universe $X$ if each $F_{j}^{m}$ is a normal fuzzy set and $F_{j}^{m}(m=$ $\left.1,2, \ldots, q_{j}\right)$ are full-overlapped in the universe $X . q_{j}$ is said to be the number of fuzzy partitions of the $j$ th input variable on $X$.

Definition 2 (see [22]). For a given fuzzy system, an overlapped-rule group (ORG) with the largest amount of rules is said to be a maximal overlapped-rule group (MORG).

Definition 3 (see [23]). Given a scalar $\gamma>0$, discrete switched system (10) is said to be robustly stable with the disturbance attenuation level $\gamma$ constraint under the $H_{\infty}$ norm if the following conditions are satisfied:

(1) When $\mathbf{w}(k) \equiv \mathbf{0}$, System (10) is asymptotically stable

(2) When $\mathbf{w}(k) \neq \mathbf{0}$, under the condition of the initial value of zero, any uncertain customers' demand meets $\|z(k)\|_{2}^{2} \leq \gamma\|\mathbf{w}(k)\|_{2}^{2}$

Property 1 (see [22]). If the input variables of a fuzzy system adopt standard fuzzy partitions, then all the rules in an ORG must be included in a MORG.

Lemma 1 (see [24]). For any real matrices $\mathbf{X}_{i j}(1 \leq i, j \leq r)$ and $\mathbf{P}>\mathbf{0}$ with appropriate dimensions, the following inequality holds:

$$
\sum_{i=1}^{r} \sum_{j=1}^{r} \sum_{p=1}^{r} \sum_{q=1}^{r} h_{i} h_{j} h_{p} h_{q} \mathbf{X}_{i j}^{\mathrm{T}} \mathbf{P} \mathbf{X}_{p q} \leq \sum_{i=1}^{r} \sum_{j=1}^{r} h_{i} h_{j} \mathbf{X}_{i j}^{\mathrm{T}} \mathbf{P} \mathbf{X}_{i j} .
$$

The FRC approach for the CLSC system will be presented in the following Theorem 1.
Theorem 1. System (10) with a certain $\gamma$ and fuzzy sets of inventories satisfying standard fuzzy partitions is robustly asymptotically stable if local common positive definite matrices $\mathbf{P}_{c}$ and $\mathbf{Q}_{e c}$ can be found in the following inequalities:

$$
\begin{aligned}
& {\left[\begin{array}{ccc}
-\overline{\overline{\mathbf{P}}} & * & * \\
\overline{\mathbf{M}}_{i i} & -\mathbf{P}_{c}^{-1} & * \\
\overline{\mathbf{N}}_{i i} & \mathbf{0} & -\mathbf{I}
\end{array}\right]<\mathbf{0 ,} i \in I_{c},} \\
& {\left[\begin{array}{ccc}
-4 \overline{\overline{\mathbf{P}}} & * & * \\
2 \overline{\overline{\mathbf{M}}}_{i j} & -\mathbf{P}_{c}^{-1} & * \\
2 \overline{\overline{\mathbf{N}}}_{i j} & \mathbf{0} & -\mathbf{I}
\end{array}\right]<\mathbf{0}, \quad i<j, i, j \in I_{c},}
\end{aligned}
$$

where

$$
\begin{aligned}
\overline{\overline{\mathbf{P}}} & =\left[\begin{array}{ccc}
\mathbf{P}_{c}-\sum_{e=1}^{g} \mathbf{Q}_{e c} & * & * \\
\mathbf{0} & \widehat{\mathbf{Q}} & * \\
\mathbf{0} & \mathbf{0} & \gamma^{2} \mathbf{I}
\end{array}\right], \\
\overline{\overline{\mathbf{M}}}_{\mathrm{ij}} & =\frac{\overline{\mathbf{M}}_{i j}+\overline{\mathbf{M}}_{j i}}{2}, \\
\widehat{\mathbf{Q}} & =\operatorname{diag}\left\{\mathbf{Q}_{1 c} \cdots\right. \\
\overline{\overline{\mathbf{N}}}_{i j} & =\frac{\left.\mathbf{Q}_{\mathrm{ec}} \cdots \mathbf{Q}_{\mathrm{gc}}\right\}}{2} .
\end{aligned}
$$

Proof. Suppose there are $f$ ORGs in System (10), $v_{d}(d=$ $1,2, \ldots, f)$ denotes the operating region of the $d$ th ORG and $L_{d}=\{$ the rule numbers involved in the $d$ th ORG $\}$.

(1) The first part of the proof:

For $\mathbf{x}(k)$ and $\mathbf{x}(k+1)$ in the same ORG, we express the local model of the $d$ th ORG as follows:

$$
\left\{\begin{array}{l}
\mathbf{x}(k+1)=\sum_{i \in L_{d}} \sum_{j \in L_{d}} h_{i} h_{j}\left[\mathbf{M}_{i j} \mathbf{x}(k)-\sum_{e=1}^{g} \overline{\mathbf{B}}_{i e} \mathbf{K}_{\mathrm{jec}} \mathbf{x}\left(k-\tau_{e}\right)+\overline{\mathbf{B}}_{w i} \mathbf{w}(k)\right], \\
z(k)=\sum_{i \in L_{d}} \sum_{j \in L_{d}} h_{i} h_{j}\left[\mathbf{N}_{i j} \mathbf{x}(k)-\sum_{e=1}^{g} \overline{\mathbf{D}}_{i e} \mathbf{K}_{\mathrm{jec}} \mathbf{x}\left(k-\tau_{e}\right)\right],
\end{array}\right.
$$


where $\mathbf{M}_{i j}=\overline{\mathbf{A}}_{i}-\overline{\mathbf{B}}_{i} \mathbf{K}_{j c}, \mathbf{N}_{i j}=\overline{\mathbf{C}}_{i}-\overline{\mathbf{D}}_{i} \mathbf{K}_{j c}$, and $\mathbf{K}_{j e c}$ denotes the state feedback gain matrix with lead times in the $c$ th MORG.

Furthermore, Model (15) is described further as follows:

$$
\left\{\begin{array}{l}
\mathbf{x}(k+1)=\sum_{i \in L_{d}} \sum_{j \in L_{d}} h_{i} h_{j} \overline{\mathbf{M}}_{i j} \overline{\mathbf{x}}(k), \\
z(k)=\sum_{i \in L_{d}} \sum_{j \in L_{d}} h_{i} h_{j} \overline{\mathbf{N}}_{i j} \overline{\mathbf{x}}(k),
\end{array}\right.
$$

where

$$
\begin{aligned}
\overline{\mathbf{M}}_{i j} & =\left[\mathbf{M}_{i j},-\overline{\mathbf{B}}_{i 1} \mathbf{K}_{j 1 c}, \ldots,-\overline{\mathbf{B}}_{i e} \mathbf{K}_{j e c}, \ldots,-\overline{\mathbf{B}}_{i g} \mathbf{K}_{j g c}, \overline{\mathbf{B}}_{w i}\right] \\
\overline{\mathbf{N}}_{i j} & =\left[\mathbf{N}_{i j},-\overline{\mathbf{D}}_{i 1} \mathbf{K}_{j 1 c}, \ldots,-\overline{\mathbf{D}}_{i e} \mathbf{K}_{j e c}, \ldots,-\overline{\mathbf{D}}_{i g} \mathbf{K}_{j g c}, \mathbf{0}\right], \\
\overline{\mathbf{x}}(k) & =\left[\mathbf{x}(k), \mathbf{x}\left(k-\tau_{1}\right), \ldots, \mathbf{x}\left(k-\tau_{e}\right), \ldots, \mathbf{x}\left(k-\tau_{g}\right), \mathbf{w}(k)\right]^{\mathrm{T}} .
\end{aligned}
$$

For System (16), a Lyapunov function is defined as follows:

$V_{d}(\mathbf{x}(k))=\mathbf{x}^{\mathrm{T}}(k) \mathbf{P}_{c} \mathbf{x}(k)+\sum_{e=1}^{g} \sum_{\xi=k-\tau_{e}}^{k-1} \mathbf{x}^{\mathrm{T}}(\xi) \mathbf{Q}_{e c} \mathbf{x}(\xi)$.

$$
\begin{aligned}
\Delta V_{d}(\mathbf{x}(k))= & V_{d}(\mathbf{x}(k+1))-V_{d}(\mathbf{x}(k)) \\
= & \mathbf{x}^{\mathrm{T}}(k+1) \mathbf{P}_{c} \mathbf{x}(k+1)-\mathbf{x}^{\mathrm{T}}(k) \mathbf{P}_{c} \mathbf{x}(k)+\sum_{e=1}^{g}\left[\mathbf{x}^{\mathrm{T}}(k) \mathbf{Q}_{e c} \mathbf{x}(k)-\mathbf{x}^{\mathrm{T}}\left(k-\tau_{e}\right) \mathbf{Q}_{e c} \mathbf{x}\left(k-\tau_{e}\right)\right] \\
= & \sum_{i \in L_{d}} \sum_{j \in L_{d}} h_{i} h_{j} \sum_{p \in L_{d}} \sum_{q \in L_{d}} h_{p} h_{q}\left[\overline{\mathbf{x}}^{\mathrm{T}}(k) \overline{\mathbf{M}}_{i j}^{\mathrm{T}} \mathbf{P}_{c} \overline{\mathbf{M}}_{p q} \overline{\mathbf{x}}(k)-\mathbf{x}^{\mathrm{T}}(k) \mathbf{P}_{c} \mathbf{x}(k)\right] \\
& +\sum_{e=1}^{g}\left[\mathbf{x}^{\mathrm{T}}(k) \mathbf{Q}_{e c} \mathbf{x}(k)-\mathbf{x}^{\mathrm{T}}\left(k-\tau_{e}\right) \mathbf{Q}_{e c} \mathbf{x}\left(k-\tau_{e}\right)\right] \\
= & \sum_{i \in L_{d}} \sum_{j \in L_{d}} h_{i} h_{j} \sum_{p \in L_{d}} \sum_{q \in L_{d}} h_{p} h_{q} \overline{\mathbf{x}}^{\mathrm{T}}(k)\left(\overline{\mathbf{M}}_{i j}^{\mathrm{T}} \mathbf{P}_{c} \overline{\mathbf{M}}_{p q}-\overline{\mathbf{P}}\right) \overline{\mathbf{x}}(k) \\
= & \sum_{i \in L_{d}} \sum_{j \in L_{d}} h_{i} h_{j} \sum_{p \in L_{d}} \sum_{q \in L_{d}} h_{p} h_{q} \overline{\mathbf{x}}^{\mathrm{T}}(k)\left[\left(\frac{\overline{\mathbf{M}}_{i j}+\overline{\mathbf{M}}_{j i}}{2}\right)^{\mathrm{T}} \mathbf{P}_{c}\left(\frac{\overline{\mathbf{M}}_{p q}+\overline{\mathbf{M}}_{q p}}{2}\right)-\overline{\mathbf{P}}\right] \overline{\mathbf{x}}(k) \\
= & \sum_{i \in L_{d}} \sum_{j \in L_{d}} h_{i} h_{j} \sum_{p \in L_{d}} \sum_{q \in L_{d}} h_{p} h_{q} \overline{\mathbf{x}}^{\mathrm{T}}(k)\left(\overline{\mathbf{M}}_{i j}^{\mathrm{T}} \mathbf{P}_{c} \overline{\overline{\mathbf{M}}}_{p q}-\overline{\mathbf{P}}\right) \overline{\mathbf{x}}(k) \\
\leq & \sum_{i \in L_{d}} \sum_{j \in L_{d}} h_{i} h_{j} \overline{\mathbf{x}}^{\mathrm{T}}(k)\left(\overline{\mathbf{M}}_{i j}^{\mathrm{T}} \mathbf{P}_{c} \overline{\overline{\mathbf{M}}}_{i j}-\overline{\mathbf{P}}\right) \overline{\mathbf{x}}(k),
\end{aligned}
$$


where

$$
\begin{aligned}
\overline{\mathbf{P}} & =\left[\begin{array}{ccc}
\mathbf{P}_{c}-\sum_{e=1}^{g} \mathbf{Q}_{e c} & * & * \\
\mathbf{0} & \widehat{\mathbf{Q}} & * \\
\mathbf{0} & \mathbf{0} & \mathbf{0}
\end{array}\right], \\
\overline{\mathbf{M}}_{\mathbf{p q}} & =\frac{\overline{\mathbf{M}}_{p q}+\overline{\mathbf{M}}_{q p}}{2}, \\
\widehat{\mathbf{Q}} & =\operatorname{diag}\left\{\mathbf{Q}_{1 c}, \mathbf{Q}_{2 c}, \ldots, \mathbf{Q}_{e c}, \ldots, \mathbf{Q}_{\mathrm{gc}}\right\}, \\
\overline{\mathbf{M}}_{\mathbf{i j}} & =\frac{\overline{\mathbf{M}}_{i j}+\overline{\mathbf{M}}_{j i}}{2} .
\end{aligned}
$$

Then, $\Delta V_{d}(\mathbf{x}(k))$ is described further as

$$
\begin{aligned}
\Delta V_{d}(\mathbf{x}(k)) \leq & \sum_{i=j, i \in L_{d}} h_{i}^{2} \overline{\mathbf{x}}^{\mathrm{T}}(k)\left[\overline{\mathbf{M}}_{i i}^{\mathrm{T}} \mathbf{P}_{c} \overline{\mathbf{M}}_{i i}-\overline{\mathbf{P}}\right] \overline{\mathbf{x}}(k) \\
& +2 \sum_{\substack{i<j \\
i \in L_{d} j \in L_{d}}} h_{i} h_{j} \overline{\mathbf{x}}^{\mathrm{T}}(k)\left[\overline{\mathbf{M}}_{i j}^{\mathrm{T}} \mathbf{P}_{c} \overline{\overline{\mathbf{M}}}_{i j}-\overline{\mathbf{P}}\right] \overline{\mathbf{x}}(k) .
\end{aligned}
$$

For $\mathbf{w}(k) \neq \mathbf{0}$, the $H_{\infty}$ performance index function is expressed as follows:

$$
J_{1}=\sum_{k=0}^{N-1}\left[z^{\mathrm{T}}(k) z(k)-\gamma^{2} \mathbf{w}^{\mathrm{T}}(k) \mathbf{w}(k)\right] .
$$

$J_{1}$ can be expressed further as

$$
\begin{aligned}
J_{1} & =\sum_{k=0}^{N-1}\left[z^{\mathrm{T}}(k) z(k)-\gamma^{2} \mathbf{w}^{\mathrm{T}}(k) \mathbf{w}(k)+\Delta V_{d}(\mathbf{x}(k))\right]-V_{d}(\mathbf{x}(N)) \\
& \leq \sum_{k=0}^{N-1}\left[z^{\mathrm{T}}(k) z(k)-\gamma^{2} \mathbf{w}^{\mathrm{T}}(k) \mathbf{w}(k)+\Delta V_{d}(\mathbf{x}(k))\right] .
\end{aligned}
$$

After Inequality (21) is introduced into (23), we have

$$
\begin{aligned}
J_{1} \leq & \sum_{k=0}^{N-1}\left\{\sum_{i=j, i \in L_{d}} h_{i}^{2} \overline{\mathbf{x}}^{\mathrm{T}}(k)\left[\overline{\mathbf{M}}_{i i}^{\mathrm{T}} \mathbf{P}_{c} \overline{\mathbf{M}}_{i i}-\overline{\overline{\mathbf{P}}}+\overline{\mathbf{N}}_{i i}^{\mathrm{T}} \overline{\mathbf{N}}_{i i}\right] \overline{\mathbf{x}}(k)\right\} \\
& +2 \sum_{k=0}^{N-1}\left\{\sum_{\substack{i<j, i \in L_{d} \\
j \in L_{d}}} h_{i} h_{j} \overline{\mathbf{x}}^{\mathrm{T}}(k)\left[\overline{\overline{\mathbf{M}}}_{i j}^{\mathrm{T}} \mathbf{P}_{c} \overline{\overline{\mathbf{M}}}_{i j}-\overline{\overline{\mathbf{P}}}+\overline{\overline{\mathbf{N}}}_{i j}^{\mathrm{T}} \overline{\mathbf{N}}_{i j}\right] \overline{\mathbf{x}}(k)\right\},
\end{aligned}
$$

where

$$
\begin{aligned}
\overline{\overline{\mathbf{P}}} & =\left[\begin{array}{ccc}
\mathbf{P}_{c}-\sum_{e=1}^{g} \mathbf{Q}_{\mathrm{ec}} & * & * \\
\mathbf{0} & \hat{\mathbf{Q}} & * \\
\mathbf{0} & \mathbf{0} & \gamma^{2} \mathbf{I}
\end{array}\right], \\
\overline{\overline{\mathbf{N}}}_{\mathrm{ij}} & =\frac{\overline{\mathbf{N}}_{i j}+\overline{\mathbf{N}}_{j i}}{2} .
\end{aligned}
$$

By the Schur complement, we have $\overline{\mathbf{M}}_{i i}^{\mathrm{T}} \mathbf{P}_{c} \overline{\mathbf{M}}_{i i}-\overline{\overline{\mathbf{P}}}+$ $\overline{\mathbf{N}}_{i i}^{\mathrm{T}} \overline{\mathbf{N}}_{i i}<\mathbf{0}$ and $\overline{\mathbf{M}}_{i j}^{\mathrm{T}} \mathbf{P}_{c} \overline{\overline{\mathbf{M}}}_{i j}-\overline{\overline{\mathbf{P}}}+\overline{\overline{\mathbf{N}}}_{i j}^{\mathrm{T}} \overline{\overline{\mathbf{N}}}_{i j}<\mathbf{0}$ which are equivalent to Inequalities (12) and (13), then $J_{1}<0$ holds, i.e., $z^{\mathrm{T}}(k) z(k)<\gamma^{2} \mathbf{w}^{\mathrm{T}}(k) \mathbf{w}(k)$; furthermore, if let $N \longrightarrow+\infty,\|z(k)\|_{2}^{2}<\gamma^{2}\|\mathbf{w}(k)\|_{2}^{2}$ holds. Therefore, CLSC system (16) is asymptotically stable under $\mathbf{w}(k) \neq \mathbf{0}$.

If $\mathbf{w}(k) \equiv \mathbf{0}$, Inequality (21) can be expressed as

$$
\begin{aligned}
\Delta V_{d}(\mathbf{x}(k)) \leq & \sum_{i=j, i \in L_{d}} h_{i}^{2} \overline{\mathbf{x}}^{\mathrm{T}}(k)\left[\overline{\mathbf{M}}_{i i}^{\mathrm{T}} \mathbf{P}_{c} \overline{\mathbf{M}}_{i i}-\overline{\overline{\mathbf{P}}}\right] \overline{\mathbf{x}}(k) \\
& +2 \sum_{\substack{i<j \\
i \in L_{d} j j L_{d}}} h_{i} h_{j} \overline{\mathbf{x}}^{\mathrm{T}}(k)\left[\overline{\mathbf{M}}_{i j}^{\mathrm{T}} \mathbf{P}_{c} \overline{\overline{\mathbf{M}}}_{i j}-\overline{\overline{\mathbf{P}}}\right] \overline{\mathbf{x}}(k) .
\end{aligned}
$$

Based on Inequalities (12) and (13), we have $\overline{\mathbf{M}}_{i i}^{\mathrm{T}} \mathbf{P}_{c} \overline{\mathbf{M}}_{i i}-\overline{\overline{\mathbf{P}}}<\mathbf{0}$ and $\overline{\overline{\mathbf{M}}}_{i j}^{\mathrm{T}} \mathbf{P}_{c} \overline{\overline{\mathbf{M}}}_{i j}-\overline{\overline{\mathbf{P}}}<\mathbf{0}$, and then $\Delta V_{d}(\mathbf{x}(k))<0$ can be obtained. Therefore, CLSC system (16) is robustly asymptotically stable under $\mathbf{w}(k) \equiv \mathbf{0}$.

(2) The second part of the proof:

For $\mathbf{x}(k)$ and $\mathbf{x}(k+1)$ in the different ORGs, we first construct the following characteristic function in any ORG:

$$
\begin{aligned}
\lambda_{d} & = \begin{cases}1, & \mathbf{x}(k) \in v_{d}, \\
0, & \mathbf{x}(k) \notin v_{d},\end{cases} \\
\sum_{d=1}^{f} \lambda_{d} & =1 .
\end{aligned}
$$

Then, the overall system of local system (16) can be expressed as

$$
\left\{\begin{array}{l}
\mathbf{x}(k+1)=\sum_{d=1}^{f} \lambda_{d}\left[\sum_{i \in L_{d}} \sum_{j \in L_{d}} h_{i} h_{j} \overline{\mathbf{M}}_{i j} \overline{\mathbf{x}}(k)\right], \\
z(k)=\sum_{d=1}^{f} \lambda_{d}\left[\sum_{i \in L_{d}} \sum_{j \in L_{d}} h_{i} h_{j} \overline{\mathbf{N}}_{i j} \overline{\mathbf{x}}(k)\right] .
\end{array}\right.
$$

After $\mathbf{P}_{m}=\sum_{d=1}^{f} \lambda_{d} \mathbf{P}_{c}$ and $\mathbf{Q}_{e m}=\sum_{d=1}^{f} \lambda_{d} \mathbf{Q}_{e c}$ are defined, we present the following Lyapunov function:

$$
\begin{aligned}
V(\mathbf{x}(k)) & =\mathbf{x}^{\mathrm{T}}(k) \mathbf{P}_{m} \mathbf{x}(k)+\sum_{e=1}^{g} \sum_{\xi=k-\tau_{e}}^{k-1} \mathbf{x}^{\mathrm{T}}(\xi) \mathbf{Q}_{e m} \mathbf{x}(\xi) \\
& =\mathbf{x}^{\mathrm{T}}(k)\left(\sum_{d=1}^{f} \lambda_{d} \mathbf{P}_{c}\right) \mathbf{x}(k)+\sum_{e=1}^{g} \sum_{\xi=k-\tau_{e}}^{k-1} \mathbf{x}^{\mathrm{T}}(\xi)\left(\sum_{d=1}^{f} \lambda_{d} \mathbf{Q}_{e c}\right) \mathbf{x}(\xi) \\
& =\sum_{d=1}^{f} \lambda_{d}\left[\mathbf{x}^{\mathrm{T}}(k) \mathbf{P}_{c} \mathbf{x}(k)+\sum_{e=1}^{g} \sum_{\xi=k-\tau_{e}}^{k-1} \mathbf{x}^{\mathrm{T}}(\xi) \mathbf{Q}_{e c} \mathbf{x}(\xi)\right] \\
& =\sum_{d=1}^{f} \lambda_{d} V_{d}(\mathbf{x}(k)) .
\end{aligned}
$$

For $\mathbf{w}(k) \neq \mathbf{0}$ in System (28), considering $J_{1}=\sum_{k=0}^{N-1}$ $\left[z^{\mathrm{T}}(k) z(k)-\gamma^{2} \mathbf{w}^{\mathrm{T}}(k) \mathbf{w}(k)\right]$, we know $J_{2}=\sum_{k=0}^{N-1}$ $\sum_{d=1}^{f} \lambda_{d}\left[z^{\mathrm{T}}(k) z(k)-\gamma^{2} \mathbf{w}^{\mathrm{T}}(k) \mathbf{w}(k)\right]$. In the same 
way, we have $J_{2}<0$, i.e., $z^{\mathrm{T}}(k) z(k)<\gamma^{2} \mathbf{w}^{\mathrm{T}}(k) \mathbf{w}(k)$; if let $N \longrightarrow+\infty,\|z(k)\|_{2}^{2}<\gamma^{2}\|\mathbf{w}(k)\|_{2}^{2}$ can be obtained. Therefore, System (28) is asymptotically stable under $\mathbf{w}(k) \neq \mathbf{0}$.

For $\mathbf{w}(k) \equiv \mathbf{0}$ in System (28), we have

$$
\begin{aligned}
\Delta V(\mathbf{x}(k)) & =V(\mathbf{x}(k+1))-V(\mathbf{x}(k)) \\
& =\sum_{d=1}^{f} \lambda_{d} V_{d}(\mathbf{x}(k+1))-\sum_{d=1}^{f} \lambda_{d} V_{d}(\mathbf{x}(k)) \\
& =\sum_{d=1}^{f} \lambda_{d}\left[V_{d}(\mathbf{x}(k+1))-V_{d}(\mathbf{x}(k))\right] \\
& =\sum_{d=1}^{f} \lambda_{d} \Delta V_{d}(\mathbf{x}(k))<0 .
\end{aligned}
$$

Therefore, System (28) under $\mathbf{w}(k) \equiv \mathbf{0}$ is asymptotically stable in any ORG.

From Proposition 1, CLSC system (10) is robustly asymptotically stable if $\mathbf{P}_{c}$ and $\mathbf{Q}_{a c}$ can be solved in Inequalities (12) and (13) Q.E.D.

In order for Inequalities (12) and (13) to be easily solvable LMIs, we transform Theorem 1 into the following Theorem 2 .

Theorem 2. System (10) with a certain $\gamma$ and fuzzy sets of inventories satisfying standard fuzzy partitions is robustly asymptotically stable if local common positive definite matrices $\mathbf{P}_{c}$ and $\mathbf{Q}_{\mathrm{ec}}$, matrices $\mathbf{K}_{i c}, \mathbf{K}_{j c}, \mathbf{K}_{i e c}$, and $\mathbf{K}_{j e c}$, and constants $\varepsilon_{i j c}>0$ and $\varepsilon_{j i c}>0$ can be found in the following Inequalities (31) and (32):

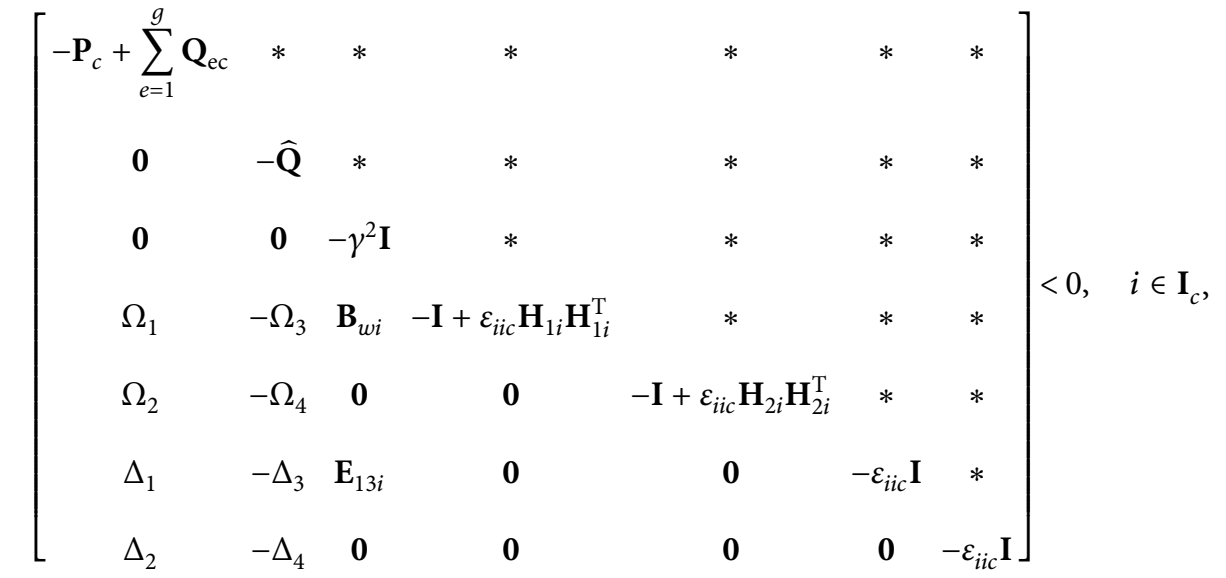

$$
\begin{aligned}
& {\left[\begin{array}{ccccccccc}
-4 \mathbf{P}_{c}+4 \sum_{e=1}^{g} \mathbf{Q}_{e c} & * & * & * & * & * & * & * & * \\
\mathbf{0} & -4 \widehat{\mathbf{Q}} & * & * & * & * & * & * & * \\
\mathbf{0} & \mathbf{0} & -4 \gamma^{2} \mathbf{I} & * & * & * & * & * & * \\
\Omega_{5} & -\Omega_{7} & \mathbf{B}_{w i}+\mathbf{B}_{w j} & \Delta_{11} & * & * & * & * & * \\
\Omega_{6} & -\Omega_{8} & \mathbf{0} & \mathbf{0} & \Delta_{12} & * & * & * & * \\
\Delta_{5} & -\Delta_{3} & \mathrm{E}_{13 i} & \mathbf{0} & \mathbf{0} & -\varepsilon_{i j c} \mathbf{I} & * & * & * \\
\Delta_{6} & -\Delta_{4} & \mathbf{0} & \mathbf{0} & \mathbf{0} & \mathbf{0} & -\varepsilon_{i j c} \mathbf{I} & * & * \\
\Delta_{7} & -\Delta_{9} & \mathrm{E}_{13 j} & \mathbf{0} & \mathbf{0} & \mathbf{0} & \mathbf{0} & -\varepsilon_{j i c} \mathbf{I} & * \\
\Delta_{8} & -\Delta_{10} & \mathbf{0} & \mathbf{0} & \mathbf{0} & \mathbf{0} & \mathbf{0} & \mathbf{0} & -\varepsilon_{j i c} \mathbf{I}
\end{array}\right]<\mathbf{0}, \quad i<j, i, j \in I_{c} \text {, }}
\end{aligned}
$$


where

$$
\begin{aligned}
\widehat{\mathbf{Q}} & =\operatorname{diag}\left\{\mathbf{Q}_{1 c}, \ldots, \mathbf{Q}_{e c}, \ldots, \mathbf{Q}_{g c}\right\}, \\
\Omega_{1} & =\mathbf{A}_{i}-\mathbf{B}_{i} \mathbf{K}_{i c}, \\
\Omega_{2} & =\mathbf{C}_{i}-\mathbf{D}_{i} \mathbf{K}_{i c}, \\
\Omega_{3} & =\mathbf{B}_{i 1} \mathbf{K}_{i 1 c}, \ldots, \mathbf{B}_{i e} \mathbf{K}_{i e c}, \ldots, \mathbf{B}_{i g} \mathbf{K}_{i g c}, \\
\Omega_{4} & =\left[\mathbf{D}_{i 1} \mathbf{K}_{i 1 c}, \ldots, \mathbf{D}_{i e} \mathbf{K}_{i e c}, \ldots, \mathbf{D}_{i g} \mathbf{K}_{i g c}\right] \\
\Omega_{5} & =\mathbf{A}_{i}-\mathbf{B}_{i} \mathbf{K}_{j c}+\mathbf{A}_{j}-\mathbf{B}_{j} \mathbf{K}_{i c}, \\
\Omega_{6} & =\mathbf{C}_{i}-\mathbf{D}_{i} \mathbf{K}_{j c}+\mathbf{C}_{j}-\mathbf{D}_{j} \mathbf{K}_{i c}, \\
\Omega_{7} & =\left[\mathbf{B}_{i 1} \mathbf{K}_{j 1 c}+\mathbf{B}_{j 1} \mathbf{K}_{i 1 c}, \ldots, \mathbf{B}_{i e} \mathbf{K}_{j e c}+\mathbf{B}_{j e} \mathbf{K}_{i e c}, \ldots, \mathbf{B}_{i g} \mathbf{K}_{j g c}+\mathbf{B}_{j g} \mathbf{K}_{i g c}\right] \\
\Omega_{8} & =\left[\mathbf{D}_{i 1} \mathbf{K}_{j 1 c}+\mathbf{D}_{j 1} \mathbf{K}_{i 1 c}, \ldots, \mathbf{D}_{i e} \mathbf{K}_{j e c}+\mathbf{D}_{j e} \mathbf{K}_{i e c}, \ldots, \mathbf{D}_{i g} \mathbf{K}_{j g c}+\mathbf{D}_{j g} \mathbf{K}_{i g c}\right], \\
\Delta_{1} & =\mathbf{E}_{11 i}-\mathbf{E}_{12 i} \mathbf{K}_{i c}, \\
\Delta_{2} & =\mathbf{E}_{21 i}-\mathbf{E}_{22 i} \mathbf{K}_{i c}, \\
\Delta_{3} & =\left[\mathbf{L}_{1 i} \mathbf{K}_{i 1 c}, \ldots, \mathbf{L}_{e i} \mathbf{K}_{i c c}, \ldots, \mathbf{L}_{g i} \mathbf{K}_{i g c}\right], \\
\Delta_{4} & =\left[\mathbf{O}_{1 i} \mathbf{K}_{i 1 c}, \ldots, \mathbf{O}_{e i} \mathbf{K}_{i e c}, \ldots, \mathbf{O}_{g i} \mathbf{K}_{i g c}\right], \\
\Delta_{5} & =\mathbf{E}_{11 i}-\mathbf{E}_{12 i} \mathbf{K}_{j c}, \\
\Delta_{6} & =\mathbf{E}_{21 i}-\mathbf{E}_{22 i} \mathbf{K}_{j c}, \\
\Delta_{7} & =\mathbf{E}_{11 j}-\mathbf{E}_{12 j} \mathbf{K}_{i c}, \\
\Delta_{8} & =\mathbf{E}_{21 j}-\mathbf{E}_{22 j} \mathbf{K}_{i c}, \\
\Delta_{9} & =\left[\mathbf{L}_{1 j} \mathbf{K}_{j i c}, \ldots, \mathbf{L}_{e j} \mathbf{K}_{j e c}, \ldots, \mathbf{L}_{g j} \mathbf{K}_{j g c}\right], \\
\Delta_{10} & =\left[\mathbf{O}_{1 j} \mathbf{K}_{j 1 c}, \ldots, \mathbf{O}_{e j} \mathbf{K}_{j e c}, \ldots, \mathbf{O}_{g j} \mathbf{K}_{j g c}\right], \\
\Delta_{11} & =-\mathbf{I}_{c}+\varepsilon_{i j c} \mathbf{H}_{1 i} \mathbf{H}_{1 i}^{\mathrm{T}}+\varepsilon_{j i c} \mathbf{H}_{1 j} \mathbf{H}_{1 j}^{\mathrm{T}}, \\
\Delta_{12} & =-\mathbf{I}+\varepsilon_{i j c} \mathbf{H}_{2 i} \mathbf{H}_{2 i}^{\mathrm{T}}+\varepsilon_{j i c} \mathbf{H}_{2 j} \mathbf{H}_{2 j}^{\mathrm{T}} . \\
&
\end{aligned}
$$

Proof. Theorem 2 can be proved in the same idea as Theorem 1. Therefore, the proof process of Theorem 2 is not shown. Q.E.D.

\section{Simulation Analysis}

We choose a type of television CLSC composed of a television manufacturer, a television retailer, and a $3 P R P$ as the simulation object to evaluate the mitigation effect of the $\mathrm{BE}$ under the FRC approach proposed in Section 4.

The television manufacturer's fuzzy membership functions and the television retailer's fuzzy membership functions are shown in Figure 2. In Figure 2, both $F_{1}^{t}\left(x_{1}(k)\right)$ and $F_{2}^{s}\left(x_{2}(k)\right)$ satisfy the conditions of standard fuzzy partition. We set $M_{1}^{1}=M_{1}^{2}=F_{1}^{1}, M_{1}^{3}=M_{1}^{4}=F_{1}^{2}, M_{2}^{1}=M_{2}^{4}=F_{2}^{1}$, and $M_{2}^{2}=M_{2}^{3}=F_{2}^{2}$.

From Figure 2, we know there are 4 fuzzy rules included in one MORG called $S$. Referring to the product-design strategies in [25], we apply 4 fuzzy rules to describe the manufacturer's production patterns and the retailer's ordering patterns for the different inventory levels as follows:
$R_{1}$ : the television manufacturer produces new televisions and remanufactures recycled televisions simultaneously. The television retailer allows return of new televisions and orders new televisions simultaneously.

$R_{2}$ : the television manufacturer produces new televisions and remanufactures recycled televisions simultaneously. The television retailer only allows return of new televisions.

$R_{3}$ : the television manufacturer only remanufactures recycled televisions. The television retailer only allows return of new televisions.

$R_{4}$ : the television manufacturer only remanufactures recycled televisions. The television retailer allows return of new televisions and orders new televisions simultaneously.

Therefore, under the different rules, the uncertain CLSC model with hybrid recycling channels and lead times can be expressed as follows: 


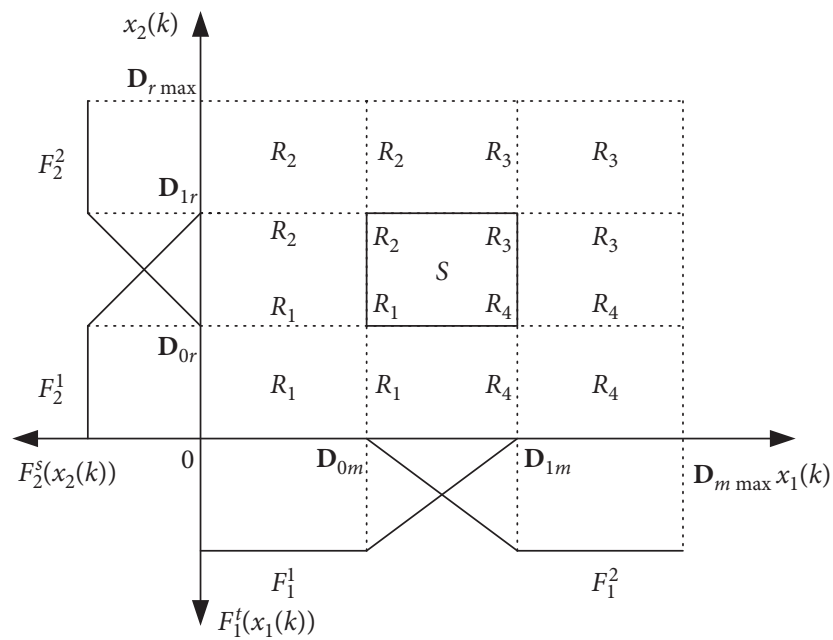

Figure 2: Fuzzy membership functions.

$$
\begin{aligned}
& R_{1}:\left\{\begin{array}{l}
x_{1}(k+1)=x_{1}(k)+u_{1}(k)+u_{1}\left(k-\tau_{1}\right)+u_{3}(k)+u_{3}\left(k-\tau_{3}\right)+(\eta+\Delta \eta) x_{4}(k)-u_{2}(k), \\
x_{2}(k+1)=x_{2}(k)+u_{2}(k)+u_{2}\left(k-\tau_{2}\right)+(\mu+\Delta \mu) x_{3}(k)-w_{1}(k), \\
x_{3}(k+1)=x_{3}(k)+w_{1}(k)-u_{3}(k)-u_{4}(k)-(\mu+\Delta \mu) x_{3}(k), \\
x_{4}(k+1)=x_{4}(k)+u_{4}(k)+u_{4}\left(k-\tau_{4}\right)-(\eta+\Delta \eta) x_{4}(k)-(\lambda+\Delta \lambda) x_{4}(k),
\end{array}\right. \\
& z(k)=\left(c_{h 1}+\Delta c_{h 1}\right) x_{1}(k)+\left(c_{h 2}+\Delta c_{h 2}\right) x_{2}(k) \\
& +\left(c_{h 3}+\Delta c_{h 3}\right) x_{4}(k)+\left(c_{n}+\Delta c_{n}\right)\left[u_{1}(k)+u_{1}\left(k-\tau_{1}\right)\right] \\
& +\left(c_{r}+\Delta c_{r}\right)\left[u_{3}(k)+u_{3}\left(k-\tau_{3}\right)+(\eta+\Delta \eta) x_{4}(k)\right] \\
& +\left(c_{m}+\Delta c_{m}\right)\left[u_{3}(k)+u_{3}\left(k-\tau_{3}\right)\right]+\left(c_{t}+\Delta c_{t}\right)\left[u_{4}(k)+u_{4}\left(k-\tau_{4}\right)\right] \\
& +\left(c_{m t}+\Delta c_{m t}\right)(\eta+\Delta \eta) x_{4}(k)+\left(c_{q}+\Delta c_{q}\right)(\mu+\Delta \mu) x_{3}(k) \\
& +\left(c_{d}+\Delta c_{d}\right)(\lambda+\Delta \lambda) x_{4}(k)+\left(c_{s}+\Delta c_{s}\right)\left[u_{2}(k)+u_{2}\left(k-\tau_{2}\right)\right] \text {, } \\
& R_{2}:\left\{\begin{array}{l}
x_{1}(k+1)=x_{1}(k)+u_{1}(k)+u_{1}\left(k-\tau_{1}\right)+u_{3}(k)+u_{3}\left(k-\tau_{3}\right)+(\eta+\Delta \eta) x_{4}(k), \\
x_{2}(k+1)=x_{2}(k)+(\mu+\Delta \mu) x_{3}(k)-w_{1}(k), \\
x_{3}(k+1)=x_{3}(k)+w_{1}(k)-u_{3}(k)-u_{4}(k)-(\mu+\Delta \mu) x_{3}(k), \\
x_{4}(k+1)=x_{4}(k)+u_{4}(k)+u_{4}\left(k-\tau_{4}\right)-(\eta+\Delta \eta) x_{4}(k)-(\lambda+\Delta \lambda) x_{4}(k),
\end{array}\right. \\
& z(k)=\left(c_{h 1}+\Delta c_{h 1}\right) x_{1}(k)+\left(c_{h 2}+\Delta c_{h 2}\right) x_{2}(k)+\left(c_{h 3}+\Delta c_{h 3}\right) x_{4}(k) \\
& +\left(c_{n}+\Delta c_{n}\right)\left[u_{1}(k)+u_{1}\left(k-\tau_{1}\right)\right]+\left(c_{r}+\Delta c_{r}\right)\left[u_{3}(k)+u_{3}\left(k-\tau_{3}\right)+(\eta+\Delta \eta) x_{4}(k)\right] \\
& +\left(c_{m}+\Delta c_{m}\right)\left[u_{3}(k)+u_{3}\left(k-\tau_{3}\right)\right]+\left(c_{t}+\Delta c_{t}\right)\left[u_{4}(k)+u_{4}\left(k-\tau_{4}\right)\right]+\left(c_{m t}+\Delta c_{m t}\right)(\eta+\Delta \eta) x_{4}(k) \\
& +\left(c_{q}+\Delta c_{q}\right)(\mu+\Delta \mu) x_{3}(k)+\left(c_{d}+\Delta c_{d}\right)(\lambda+\Delta \lambda) x_{4}(k), \\
& R_{3}:\left\{\begin{array}{l}
x_{1}(k+1)=x_{1}(k)+u_{3}(k)+u_{3}\left(k-\tau_{3}\right)+(\eta+\Delta \eta) x_{4}(k), \\
x_{2}(k+1)=x_{2}(k)+(\mu+\Delta \mu) x_{3}(k)-w_{1}(k), \\
x_{3}(k+1)=x_{3}(k)+w_{1}(k)-u_{3}(k)-u_{4}(k)-(\mu+\Delta \mu) x_{3}(k), \\
x_{4}(k+1)=x_{4}(k)+u_{4}(k)+u_{4}\left(k-\tau_{4}\right)-(\eta+\Delta \eta) x_{4}(k)-(\lambda+\Delta \lambda) x_{4}(k),
\end{array}\right. \\
& z(k)=\left(c_{h 1}+\Delta c_{h 1}\right) x_{1}(k)+\left(c_{h 2}+\Delta c_{h 2}\right) x_{2}(k)+\left(c_{h 3}+\Delta c_{h 3}\right) x_{4}(k) \\
& +\left(c_{r}+\Delta c_{r}\right)\left[u_{3}(k)+u_{3}\left(k-\tau_{3}\right)+(\eta+\Delta \eta) x_{4}(k)\right] \\
& +\left(c_{m}+\Delta c_{m}\right)\left[u_{3}(k)+u_{3}\left(k-\tau_{3}\right)\right]+\left(c_{t}+\Delta c_{t}\right)\left[u_{4}(k)+u_{4}\left(k-\tau_{4}\right)\right] \\
& +\left(c_{m t}+\Delta c_{m t}\right)(\eta+\Delta \eta) x_{4}(k)+\left(c_{q}+\Delta c_{q}\right)(\mu+\Delta \mu) x_{3}(k) \\
& +\left(c_{d}+\Delta c_{d}\right)(\lambda+\Delta \lambda) x_{4}(k) \text {, } \\
& R_{4}:\left\{\begin{array}{l}
x_{1}(k+1)=x_{1}(k)+u_{3}(k)+u_{3}\left(k-\tau_{3}\right)+(\eta+\Delta \eta) x_{4}(k)-u_{2}(k), \\
x_{2}(k+1)=x_{2}(k)+u_{2}(k)+u_{2}\left(k-\tau_{2}\right)+(\mu+\Delta \mu) x_{3}(k)-w_{1}(k), \\
x_{3}(k+1)=x_{3}(k)+w_{1}(k)-u_{3}(k)-u_{4}(k)-(\mu+\Delta \mu) x_{3}(k), \\
x_{4}(k+1)=x_{4}(k)+u_{4}(k)+u_{4}\left(k-\tau_{4}\right)-(\eta+\Delta \eta) x_{4}(k)-(\lambda+\Delta \lambda) x_{4}(k) .
\end{array}\right.
\end{aligned}
$$




$$
\begin{aligned}
z(k)= & \left(c_{h 1}+\Delta c_{h 1}\right) x_{1}(k)+\left(c_{h 2}+\Delta c_{h 2}\right) x_{2}(k)+\left(c_{h 3}+\Delta c_{h 3}\right) x_{4}(k) \\
& +\left(c_{r}+\Delta c_{r}\right)\left[u_{3}(k)+u_{3}\left(k-\tau_{3}\right)+(\eta+\Delta \eta) x_{4}(k)\right] \\
& +\left(c_{m}+\Delta c_{m}\right)\left[u_{3}(k)+u_{3}\left(k-\tau_{3}\right)\right]+\left(c_{t}+\Delta c_{t}\right)\left[u_{4}(k)+u_{4}\left(k-\tau_{4}\right)\right] \\
& +\left(c_{m t}+\Delta c_{m t}\right)(\eta+\Delta \eta) x_{4}(k) \\
& +\left(c_{q}+\Delta c_{q}\right)(\mu+\Delta \mu) x_{3}(k)+\left(c_{d}+\Delta c_{d}\right)(\lambda+\Delta \lambda) x_{4}(k) \\
& +\left(c_{s}+\Delta c_{s}\right)\left[u_{2}(k)+u_{2}\left(k-\tau_{2}\right)\right]
\end{aligned}
$$

Furthermore, the fuzzy CLSC model can be obtained as follows:

$R_{1}$ : If $x_{1}$ is $M_{1}^{1}$ and $x_{2}$ is $M_{2}^{1}$, then

$$
\left\{\begin{array}{l}
\mathbf{x}(k+1)=h_{1}\left[\left(\mathbf{A}_{1}+\Delta \mathbf{A}_{1}\right) \mathbf{x}(k)+\left(\mathbf{B}_{1}+\Delta \mathbf{B}_{1}\right) \mathbf{u}(k)+\sum_{e=1}^{4}\left(\mathbf{B}_{1 e}+\Delta \mathbf{B}_{1 e}\right) \mathbf{u}\left(k-\tau_{e}\right)+\left(\mathbf{B}_{w 1}+\Delta \mathbf{B}_{w 1}\right) \mathbf{w}(k)\right], \\
z(k)=h_{1}\left[\left(\mathbf{C}_{1}+\Delta \mathbf{C}_{1}\right) \mathbf{x}(k)+\left(\mathbf{D}_{1}+\Delta \mathbf{D}_{1}\right) \mathbf{u}(k)+\sum_{e=1}^{4}\left(\mathbf{D}_{1 e}+\Delta \mathbf{D}_{1 e}\right) \mathbf{u}\left(k-\tau_{e}\right)\right]
\end{array}\right.
$$

$R_{2}$ : If $x_{1}$ is $M_{1}^{2}$ and $x_{2}$ is $M_{2}^{2}$, then

$$
\left\{\begin{array}{l}
\mathbf{x}(k+1)=h_{2}\left[\left(\mathbf{A}_{2}+\Delta \mathbf{A}_{2}\right) \mathbf{x}(k)+\left(\mathbf{B}_{2}+\Delta \mathbf{B}_{2}\right) \mathbf{u}(k)+\sum_{e=1}^{4}\left(\mathbf{B}_{2 e}+\Delta \mathbf{B}_{2 e}\right) \mathbf{u}\left(k-\tau_{e}\right)+\left(\mathbf{B}_{w 2}+\Delta \mathbf{B}_{w 2}\right) \mathbf{w}(k)\right] \\
z(k)=h_{2}\left[\left(\mathbf{C}_{2}+\Delta \mathbf{C}_{2}\right) \mathbf{x}(k)+\left(\mathbf{D}_{2}+\Delta \mathbf{D}_{2}\right) \mathbf{u}(k)+\sum_{e=1}^{4}\left(\mathbf{D}_{2 e}+\Delta \mathbf{D}_{2 e}\right) \mathbf{u}\left(k-\tau_{e}\right)\right] .
\end{array}\right.
$$

$R_{3}$ : If $x_{1}$ is $M_{1}^{3}$ and $x_{2}$ is $M_{2}^{3}$, then

$$
\left\{\begin{array}{l}
\mathbf{x}(k+1)=h_{3}\left[\left(\mathbf{A}_{3}+\Delta \mathbf{A}_{3}\right) \mathbf{x}(k)+\left(\mathbf{B}_{3}+\Delta \mathbf{B}_{3}\right) \mathbf{u}(k)+\sum_{e=1}^{4}\left(\mathbf{B}_{3 e}+\Delta \mathbf{B}_{3 e}\right) \mathbf{u}\left(k-\tau_{e}\right)+\left(\mathbf{B}_{w 3}+\Delta \mathbf{B}_{w 3}\right) \mathbf{w}(k)\right], \\
z(k)=h_{3}\left[\left(\mathbf{C}_{3}+\Delta \mathbf{C}_{3}\right) \mathbf{x}(k)+\left(\mathbf{D}_{3}+\Delta \mathbf{D}_{3}\right) \mathbf{u}(k)+\sum_{e=1}^{4}\left(\mathbf{D}_{3 e}+\Delta \mathbf{D}_{3 e}\right) \mathbf{u}\left(k-\tau_{e}\right)\right] .
\end{array}\right.
$$

$R_{4}$ : If $x_{1}$ is $M_{1}^{4}$ and $x_{2}$ is $M_{2}^{4}$, then

$$
\left\{\begin{array}{l}
\mathbf{x}(k+1)=h_{4}\left[\left(\mathbf{A}_{4}+\Delta \mathbf{A}_{4}\right) \mathbf{x}(k)+\left(\mathbf{B}_{4}+\Delta \mathbf{B}_{4}\right) \mathbf{u}(k)+\sum_{e=1}^{4}\left(\mathbf{B}_{4 e}+\Delta \mathbf{B}_{4 e}\right) \mathbf{u}\left(k-\tau_{e}\right)+\left(\mathbf{B}_{w 4}+\Delta \mathbf{B}_{w 4}\right) \mathbf{w}(k)\right] \\
z(k)=h_{4}\left[\left(\mathbf{C}_{4}+\Delta \mathbf{C}_{4}\right) \mathbf{x}(k)+\left(\mathbf{D}_{4}+\Delta \mathbf{D}_{4}\right) \mathbf{u}(k)+\sum_{e=1}^{4}\left(\mathbf{D}_{4 e}+\Delta \mathbf{D}_{4 e}\right) \mathbf{u}\left(k-\tau_{e}\right)\right]
\end{array}\right.
$$

According to the Takagi-Sugeno fuzzy models mentioned above, we design the following fuzzy feedback controller.

$$
\left\{\begin{array}{l}
\mathbf{u}(k)=-\sum_{i=1}^{4} h_{i} \mathbf{K}_{i 1} \mathbf{x}(k), \\
\mathbf{u}\left(k-\tau_{1}\right)=-\sum_{i=1}^{4} h_{i} \mathbf{K}_{i 11} \mathbf{x}\left(k-\tau_{1}\right), \\
\mathbf{u}\left(k-\tau_{2}\right)=-\sum_{i=1}^{4} h_{i} \mathbf{K}_{i 21} \mathbf{x}\left(k-\tau_{2}\right), \\
\mathbf{u}\left(k-\tau_{3}\right)=-\sum_{i=1}^{4} h_{i} \mathbf{K}_{i 31} \mathbf{x}\left(k-\tau_{3}\right), \\
\mathbf{u}\left(k-\tau_{4}\right)=-\sum_{i=1}^{4} h_{i} \mathbf{K}_{i 41} \mathbf{x}\left(k-\tau_{4}\right) .
\end{array}\right.
$$


Considering the actual operation situation of the television CLSC, we set $D_{0 m}=120, D_{1 m}=160, D_{0 r}=110$, and $D_{1 r}=155\left(\times 10^{3}\right.$ sets); $c_{h 1}=0.015, c_{h 2}=0.020, c_{h 3}=0.015$, $c_{n}=0.150, \quad c_{r}=0.005, \quad c_{m}=0.100, \quad c_{m t}=0.100, \quad c_{t}=0.095$, $c_{q}=0.020, \quad c_{d}=0.007, \quad c_{s}=0.180 \quad\left(\times 10^{3}\right.$ Yuan); $\eta=0.98$, $\mu=0.01$, and $\lambda=0.02$.

$$
\begin{aligned}
& \mathbf{A}_{1}=\mathbf{A}_{2}=\mathbf{A}_{3}=\mathbf{A}_{4}=\left[\begin{array}{cccc}
1 & 0 & 0 & 0.98 \\
0 & 1 & 0.01 & 0 \\
0 & 0 & 0.99 & 0 \\
0 & 0 & 0 & 0
\end{array}\right], \\
& \mathbf{B}_{1}=\left[\begin{array}{cccc}
1 & -1 & 1 & 0 \\
0 & 1 & 0 & 0 \\
0 & 0 & -1 & -1 \\
0 & 0 & 0 & 1
\end{array}\right], \\
& \mathbf{B}_{2}=\left[\begin{array}{cccc}
1 & 0 & 1 & 0 \\
0 & 0 & 0 & 0 \\
0 & 0 & -1 & -1 \\
0 & 0 & 0 & 1
\end{array}\right], \\
& \mathbf{B}_{3}=\left[\begin{array}{cccc}
0 & 0 & 1 & 0 \\
0 & 0 & 0 & 0 \\
0 & 0 & -1 & -1 \\
0 & 0 & 0 & 1
\end{array}\right], \\
& \mathbf{B}_{4}=\left[\begin{array}{cccc}
0 & -1 & 1 & 0 \\
0 & 1 & 0 & 0 \\
0 & 0 & -1 & -1 \\
0 & 0 & 0 & 1
\end{array}\right], \\
& \mathbf{B}_{11}=\mathbf{B}_{21}=\left[\begin{array}{llll}
1 & 0 & 0 & 0 \\
0 & 0 & 0 & 0 \\
0 & 0 & 0 & 0 \\
0 & 0 & 0 & 0
\end{array}\right] \text {, } \\
& \mathbf{B}_{31}=\mathbf{B}_{41}=\left[\begin{array}{llll}
0 & 0 & 0 & 0 \\
0 & 0 & 0 & 0 \\
0 & 0 & 0 & 0 \\
0 & 0 & 0 & 0
\end{array}\right], \\
& \mathbf{B}_{12}=\mathbf{B}_{42}=\left[\begin{array}{llll}
0 & 0 & 0 & 0 \\
0 & 1 & 0 & 0 \\
0 & 0 & 0 & 0 \\
0 & 0 & 0 & 0
\end{array}\right], \\
& \mathbf{B}_{22}=\mathbf{B}_{32}=\left[\begin{array}{llll}
0 & 0 & 0 & 0 \\
0 & 0 & 0 & 0 \\
0 & 0 & 0 & 0 \\
0 & 0 & 0 & 0
\end{array}\right], \\
& \mathbf{B}_{13}=\mathbf{B}_{23}=\mathbf{B}_{33}=\mathbf{B}_{43}=\left[\begin{array}{llll}
0 & 0 & 1 & 0 \\
0 & 0 & 0 & 0 \\
0 & 0 & 0 & 0 \\
0 & 0 & 0 & 0
\end{array}\right] \text {, } \\
& \mathbf{B}_{14}=\mathbf{B}_{24}=\mathbf{B}_{34}=\mathbf{B}_{44}=\left[\begin{array}{llll}
0 & 0 & 0 & 0 \\
0 & 0 & 0 & 0 \\
0 & 0 & 0 & 0 \\
0 & 0 & 0 & 1
\end{array}\right], \\
& \mathbf{B}_{w 1}=\mathbf{B}_{w 2}=\mathbf{B}_{w 3}=\mathbf{B}_{w 4}=\left[\begin{array}{cccc}
0 & 0 & 0 & 0 \\
-1 & 0 & 0 & 0 \\
1 & 0 & 0 & 0 \\
0 & 0 & 0 & 0
\end{array}\right],
\end{aligned}
$$

$$
\mathbf{C}_{1}=\mathbf{C}_{2}=\mathbf{C}_{3}=\mathbf{C}_{4}=\left[\begin{array}{llll}
c_{h 1} & c_{h 2} & \mu c_{q} & c_{h 3}+\eta c_{r}+\eta c_{m t}+\lambda c_{d}
\end{array}\right],
$$$$
\mathbf{D}_{1}=\left[\begin{array}{llll}
c_{n} & c_{s} & c_{r}+c_{m} & c_{t}
\end{array}\right] \text {, }
$$$$
\mathbf{D}_{2}=\left[\begin{array}{llll}
c_{n} & 0 & c_{r}+c_{m} & c_{t}
\end{array}\right] \text {, }
$$$$
\mathbf{D}_{3}=\left[\begin{array}{llll}
0 & 0 & c_{r}+c_{m} & c_{t}
\end{array}\right] \text {, }
$$$$
\mathbf{D}_{4}=\left[\begin{array}{lllll}
0 & c_{s} & c_{r}+c_{m} & c_{t}
\end{array}\right] \text {, }
$$$$
\mathbf{D}_{11}=\mathbf{D}_{21}=\left[\begin{array}{llll}
c_{n} & 0 & 0 & 0
\end{array}\right] \text {, }
$$$$
\mathbf{D}_{31}=\mathbf{D}_{41}=\left[\begin{array}{llll}
0 & 0 & 0 & 0
\end{array}\right] \text {, }
$$$$
\mathbf{D}_{12}=\mathbf{D}_{42}=\left[\begin{array}{llll}
0 & c_{s} & 0 & 0
\end{array}\right] \text {, }
$$$$
\mathbf{D}_{22}=\mathbf{D}_{32}=\left[\begin{array}{llll}
0 & 0 & 0 & 0
\end{array}\right] \text {, }
$$$$
\mathbf{D}_{13}=\mathbf{D}_{23}=\mathbf{D}_{33}=\mathbf{D}_{43}=\left[\begin{array}{llll}
0 & 0 & c_{r}+c_{m} & 0
\end{array}\right] \text {, }
$$$$
\mathbf{D}_{14}=\mathbf{D}_{24}=\mathbf{D}_{34}=\mathbf{D}_{44}=\left[\begin{array}{llll}
0 & 0 & 0 & c_{t}
\end{array}\right] \text {, }
$$$$
\mathbf{E}_{11 i}=\left[\begin{array}{cccc}
0 & 0 & 0 & 0.02 \\
0 & 0 & 0.02 & 0 \\
0 & 0 & -0.02 & 0 \\
0 & 0 & 0 & -0.03
\end{array}\right] \text {, }
$$$$
\mathbf{E}_{12 i}=\mathbf{E}_{13 i}=\mathbf{0} \text {, }
$$$$
\mathbf{L}_{1 i}=\mathbf{L}_{2 i}=\mathbf{L}_{3 i}=\mathbf{L}_{4 i}=\mathbf{0} \text {, }
$$$$
\mathbf{E}_{21 i}=\left[\begin{array}{llll}
0.004 & 0.004 & 0.002 & 0.007
\end{array}\right],
$$$$
\mathbf{E}_{221}=\left[\begin{array}{llll}
0.003 & 0.002 & 0.015 & 0.015
\end{array}\right],
$$$$
\mathbf{E}_{222}=\left[\begin{array}{llll}
0.003 & 0 & 0.015 & 0.015
\end{array}\right],
$$$$
\mathbf{E}_{223}=\left[\begin{array}{llll}
0 & 0 & 0.015 & 0.015
\end{array}\right] \text {, }
$$$$
\mathbf{E}_{224}=\left[\begin{array}{llll}
0 & 0.002 & 0.015 & 0.015
\end{array}\right],
$$$$
\mathbf{O}_{11}=\mathbf{O}_{21}=\left[\begin{array}{llll}
0.001 & 0 & 0 & 0
\end{array}\right] \text {, }
$$$$
\mathbf{O}_{31}=\mathbf{O}_{41}=\left[\begin{array}{llll}
0 & 0 & 0 & 0
\end{array}\right] \text {, }
$$$$
\mathbf{O}_{12}=\mathbf{O}_{42}=\left[\begin{array}{llll}
0 & 0.002 & 0 & 0
\end{array}\right] \text {, }
$$$$
\mathbf{O}_{22}=\mathbf{O}_{32}=\left[\begin{array}{llll}
0 & 0 & 0 & 0
\end{array}\right] \text {, }
$$$$
\mathbf{O}_{13}=\mathbf{O}_{23}=\mathbf{O}_{33}=\mathbf{O}_{43}=\left[\begin{array}{llll}
0 & 0 & 0.002 & 0
\end{array}\right] \text {, }
$$$$
\mathbf{O}_{14}=\mathbf{O}_{24}=\mathbf{O}_{34}=\mathbf{O}_{44}=\left[\begin{array}{llll}
0 & 0 & 0 & 0.001
\end{array}\right] \text {, }
$$$$
\mathbf{H}_{1 i}=0.1 \text {, }
$$$$
\mathbf{H}_{2 i}=0.2 \text {, }
$$$$
\mathbf{F}_{1 i}=\mathbf{F}_{2 i}=\sin (k),
$$

$$
\begin{aligned}
& (i=1,2,3,4), \\
& \gamma=0.5 .
\end{aligned}
$$




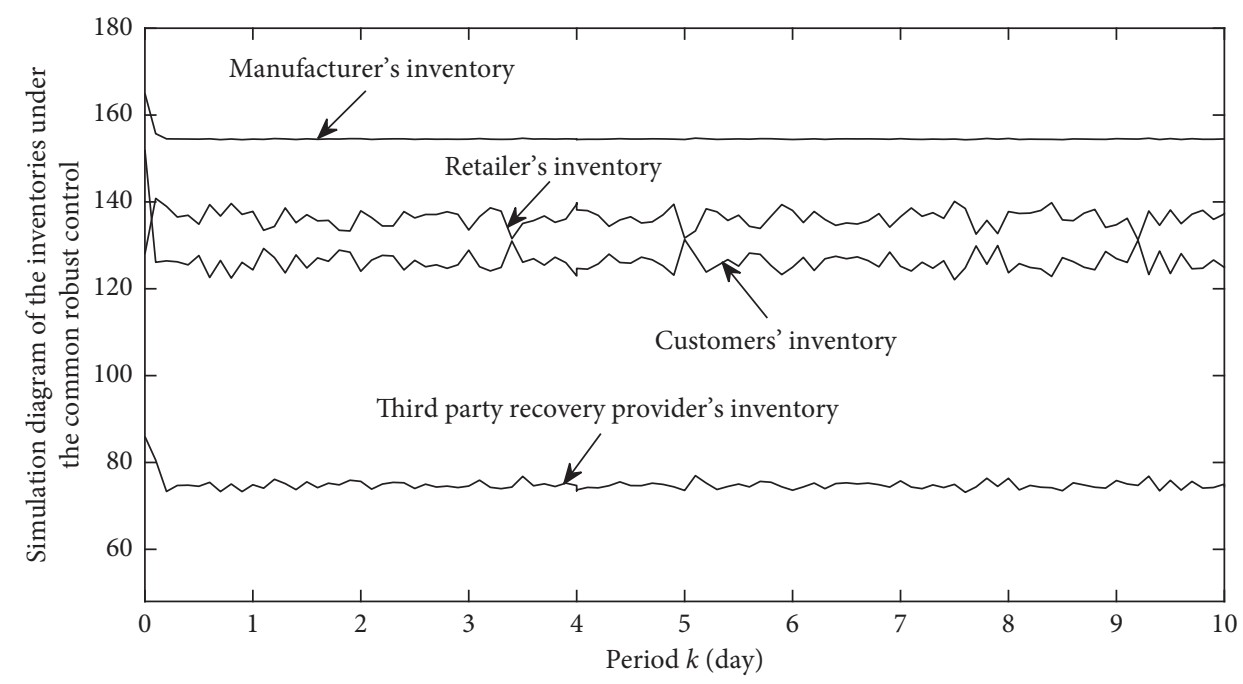

Figure 3: Simulation diagram of the inventories under CRC (unit: $\times 10^{3}$ sets).

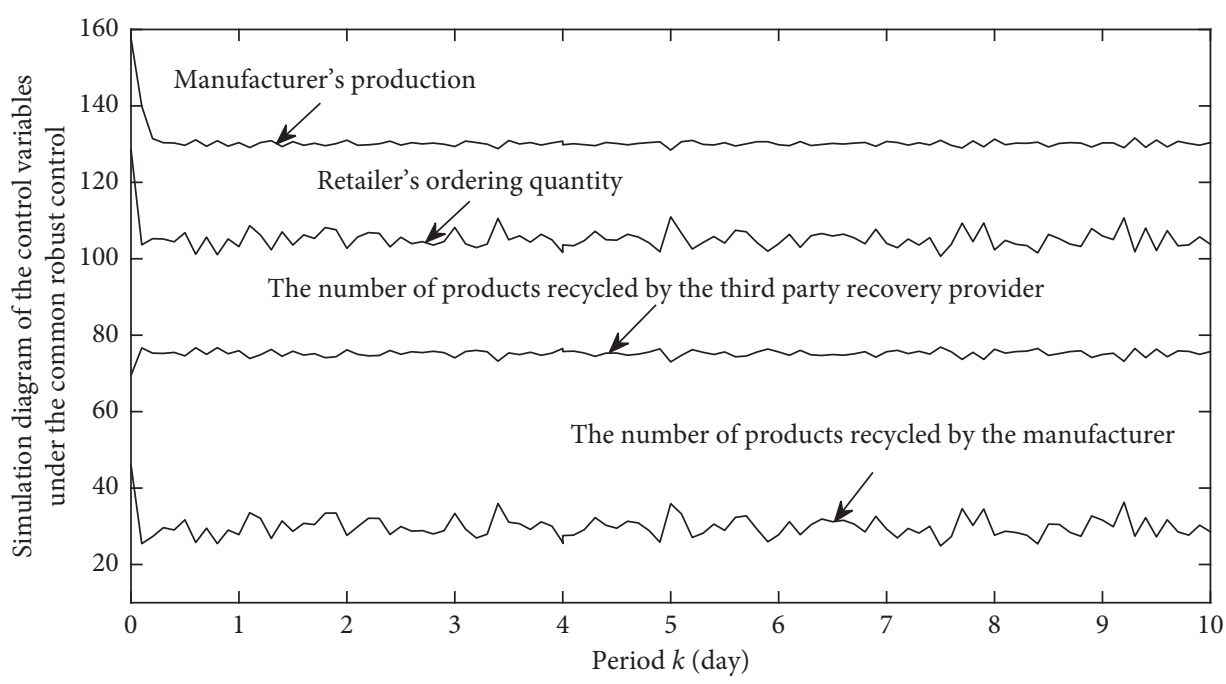

FIgURe 4: Simulation diagram of the control variables under CRC (unit: $\times 10^{3}$ sets).

The FRC approach proposed in Section 4 can guarantee the television CLSC system to be robustly stable after the following results, which meet Conditions (31) and (32) in Theorem 2, are obtained.

$$
\begin{aligned}
& \mathbf{P}_{1}=\left[\begin{array}{cccc}
121.8898 & 0.0028 & 0.1511 & 0.1485 \\
0.0028 & 122.7989 & 0.0013 & -0.0113 \\
0.1511 & 0.0013 & 121.8829 & 0.1509 \\
0.1485 & -0.0113 & 0.1509 & 121.8811
\end{array}\right], \\
& \mathbf{Q}_{11}=\mathbf{Q}_{21}=\mathbf{Q}_{31}=\mathbf{Q}_{41}=\left[\begin{array}{rrrr}
24.3455 & -0.0000 & -0.0006 & -0.0006 \\
-0.0000 & 24.3419 & -0.0000 & 0.0000 \\
-0.0006 & -0.0000 & 24.3455 & -0.0006 \\
-0.0006 & 0.0000 & -0.0006 & 24.3455
\end{array}\right],
\end{aligned}
$$




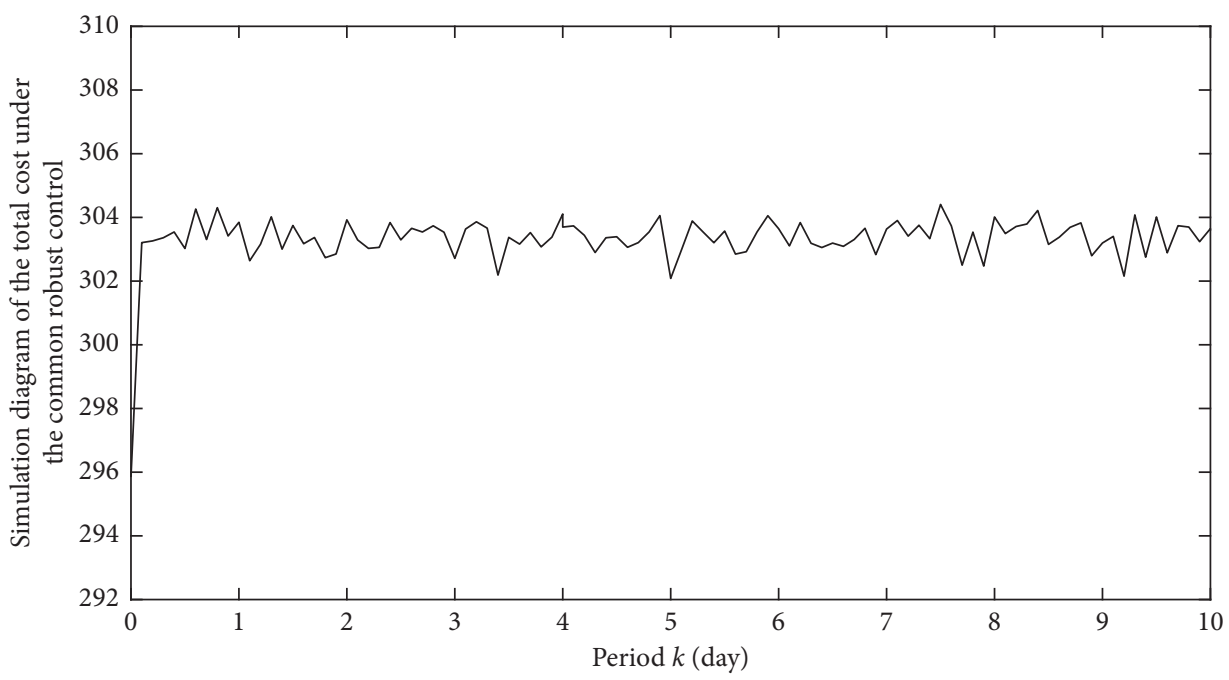

FIgURE 5: Simulation diagram of the total cost under CRC (unit: $\times 10^{5}$ Yuan).

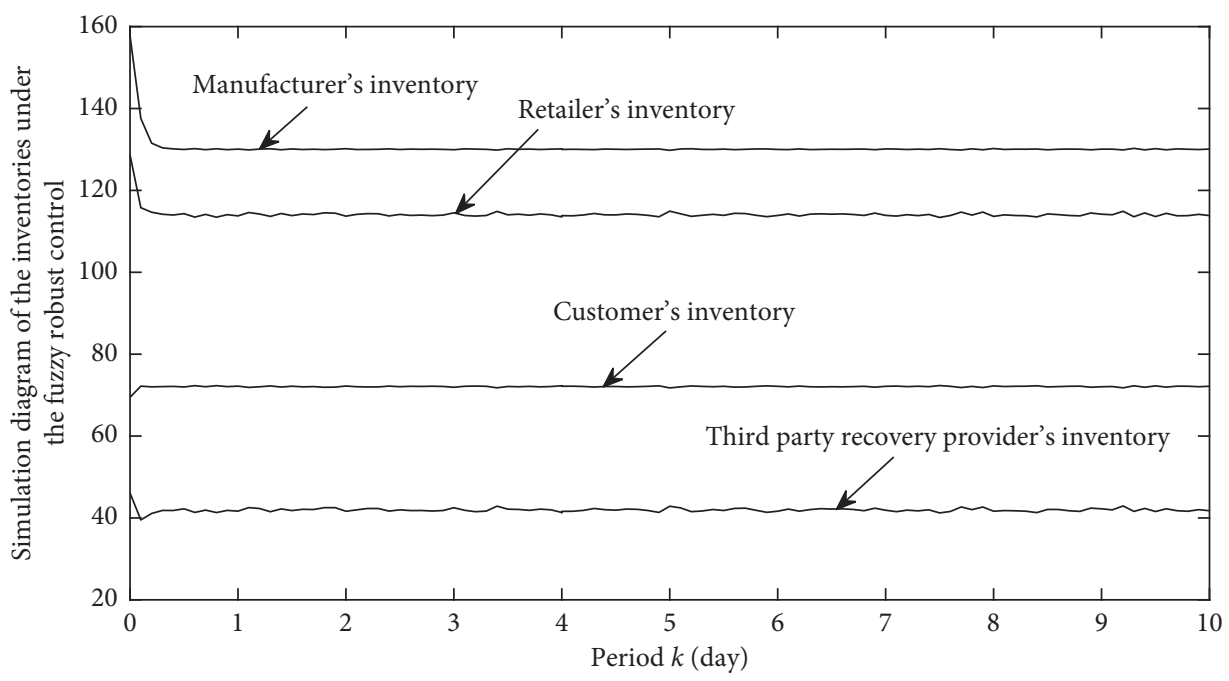

FIgURE 6: Simulation diagram of the inventories under FRC (unit: $\times 10^{3}$ sets).

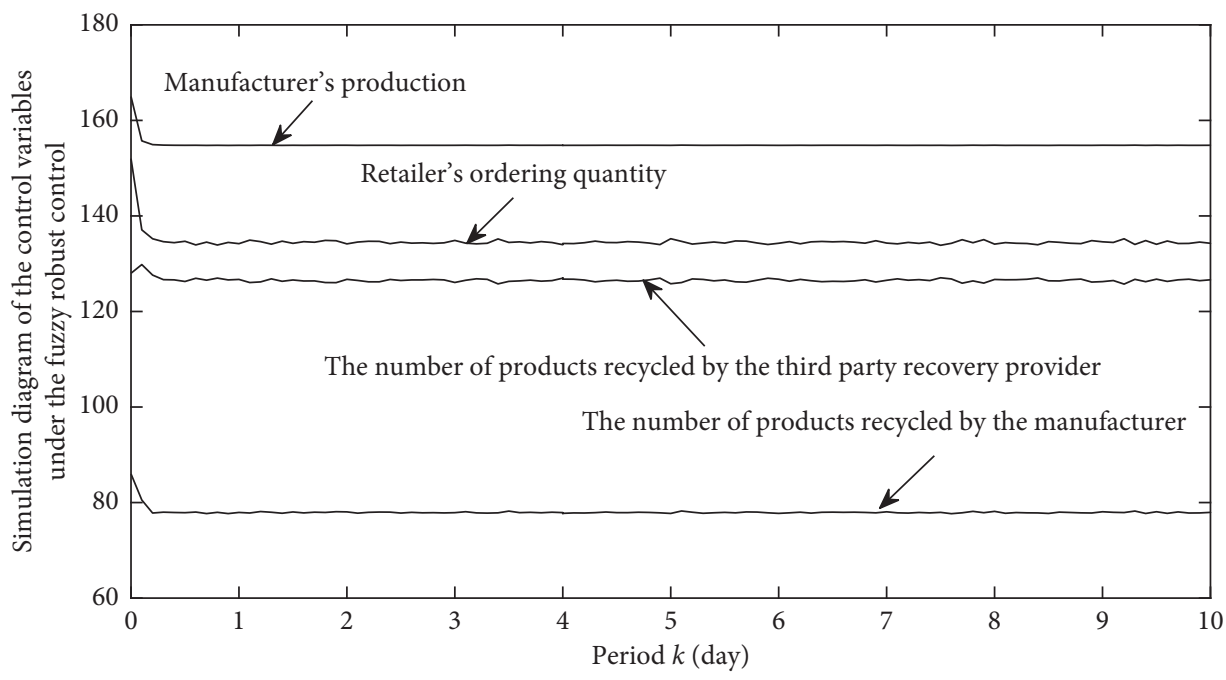

FIGURE 7: Simulation diagram of the control variables under FRC (unit: $\times 10^{3}$ sets). 


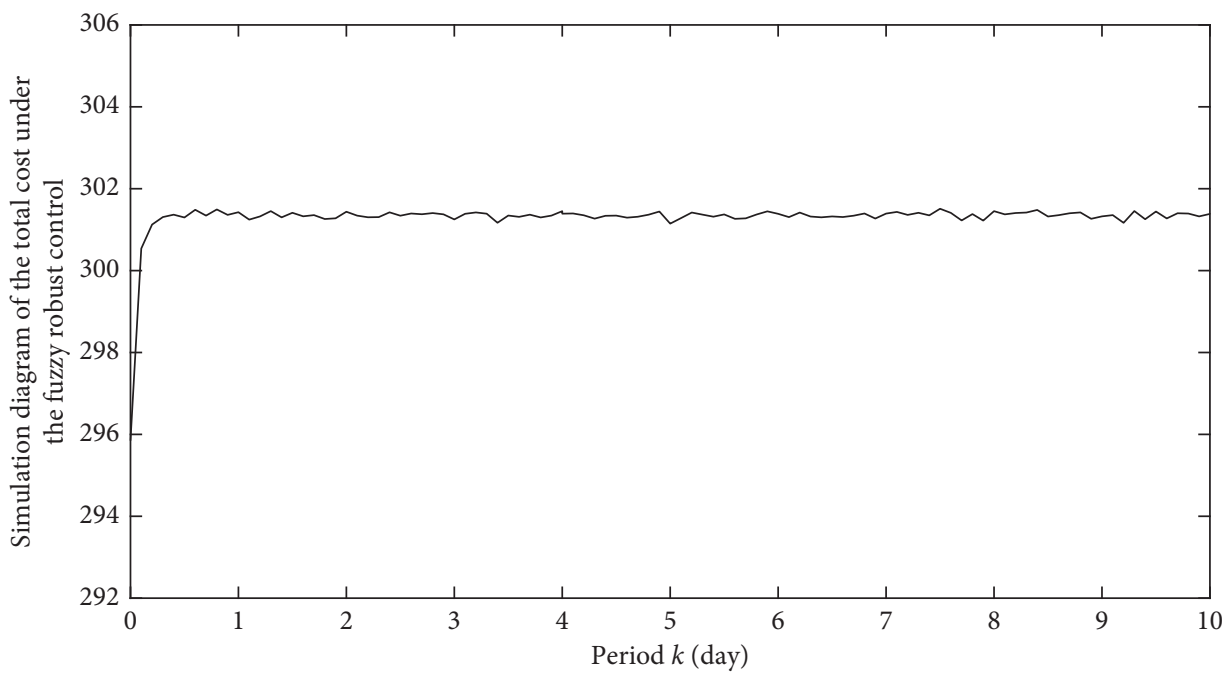

FIGURE 8: Simulation diagram of the total cost under FRC (unit: $\times 10^{5}$ Yuan).

Then, we will perform the simulation tests to compare the mitigation effect of the $\mathrm{BE}$ between the proposed FRC approach and the CRC approach. Set the initial values as follows: $x_{1}(0)=14, x_{2}(0)=12, x_{3}(0)=8$, and $x_{4}(0)=6$ (unit: $\times 10^{3}$ sets). Set the nominal values as follows: $\vec{x}_{1}=155, \vec{x}_{2}=140, \vec{x}_{3}=120$, and $\vec{x}_{4}=80$ (unit: $\times 10^{3}$ sets); $\vec{u}_{1}=130, \vec{u}_{2}=120, \vec{u}_{3}=50$, and $\vec{u}_{4}=70$ (unit: $\times 10^{3}$ sets); we let $\tau_{1}=\tau_{2}=\tau_{3}=\tau_{4}=4$ (unit: day), and the customers' demand meets the normal distribution $N\left(6,0.3^{2}\right)$. Then, the simulation results are shown in Figures 3-8, in which Figures 3-5 are the simulation diagrams under CRC, and Figures 6-8 are the simulation diagrams under FRC.

As shown in Figures 3-5, by utilizing the CRC approach to mitigate the $\mathrm{BE}$ of the uncertain CLSC with hybrid recycling channels and lead times, there are larger fluctuations for the variables of the CLSC system, which result in the worse mitigation level of the $\mathrm{BE}$ and the higher total cost of the CLSC. On the contrary, it can be seen from Figures 6-8 that the FRC approach proposed in this paper can mitigate the BE more effectively and make the CLSC system to be robustly stable. Also, the fluctuation ranges of all variables under the FRC approach are less that those under the CRC approach. Especially, compared with the CRC approach, the FRC approach realizes the total cost of the CLSC system in the stable and lower levels. Therefore, for the mitigation of the $\mathrm{BE}$ caused by uncertainties and lead times, the FRC approach proposed in this paper can more effectively guarantee the interests of enterprises in the supply chain and ensure the long-term and stable development of the CLSC.

\section{Conclusions}

$\mathrm{BE}$ is a universal phenomenon in the operation process of the supply chain system. Therefore, the mitigation of the BE has become one of the most focused and urgent issues for enterprises in the supply chain. In this paper, we have set up an uncertain model of the CLSC with hybrid recycling channels and lead times. To implement the flexible switching in the CLSC system, the aforementioned model is transformed into a CLSC fuzzy model. Then, the manufacturer's production patterns and the retailer's ordering patterns are put forward according to their respective inventory levels. A new FRC approach is proposed to mitigate the $\mathrm{BE}$ of the uncertain CLSC with lead times. Simulation results show that this approach can effectively mitigate the impacts of uncertainties and lead times on the BE of the CLSC and guarantee the CLSC system in a robust stable state, which will reduce the loss of the CLSC caused by the BE. It is known from this paper and [19] that the FRC approach is an effective approach to mitigate the $\mathrm{BE}$ and can improve the mitigation effect of the CRC approach. For the future research directions, one is how to coordinate the cost sharing among the manufacturer, the retailer, and the 3PRP; another is that the FRC approach is combined with other approaches to more effectively mitigate the $\mathrm{BE}$.
Abbreviations
$x_{1}(k)$ :
$x_{2}(k)$ :
$x_{3}(k)$ :
$x_{4}(k):$
$u_{1}(k)$ :
$u_{2}(k):$
$u_{3}(k)$ :
$u_{4}(k)$ :
$w_{1}(k)$ :
$\tau_{1}$ :
$\tau_{2}$ :
$\tau_{3}:$
Manufacturer's inventory at period $k$
Retailer's inventory at period $k$
Customers' inventory at period $k$
3PRP's inventory at period $k$
Manufacturer's production variable at period $k$
Retailer's ordering variable at period $k$
Manufacturer's used products recycling
variable at period $k$
3PRP's used products recycling variable at
period $k$
Customers' demand at period $k$
Manufacturer's production lead time
Retailer's ordering lead time
Lead time of the used products recycled by the manufacturer
$\tau_{4}: \quad$ Lead time of the used products recycled by 3PRP
$\eta$ : $\quad$ Remanufacturing rate 
$\Delta \eta: \quad$ Uncertainty of $\eta$

$\mu$ : $\quad$ Return rate of the new products

$\Delta \mu: \quad$ Uncertainty of $\mu$

$\lambda: \quad$ Disposal rate

$\Delta \lambda: \quad$ Uncertainty of $\lambda 0 \leq \eta, \mu, \lambda \leq 1, \eta+\lambda=1$

$z(k): \quad$ Total cost of the CLSC at period $k$

$c_{h 1}$ : $\quad$ Manufacturer's unit inventory cost

$\Delta c_{h 1}: \quad$ Uncertainty of $c_{h 1}$

$c_{h 2}: \quad$ Retailer's unit inventory cost

$\Delta c_{h 2}: \quad$ Uncertainty of $c_{h 2}$

$c_{h 3}: \quad$ 3PRP's unit inventory cost

$\Delta c_{h 3}: \quad$ Uncertainty of $c_{h 3}$

$c_{n}$ : $\quad$ Unit production cost of the new product

$\Delta c_{n}: \quad$ Uncertainty of $c_{n}$

$c_{r}: \quad$ Unit remanufacturing cost

$\Delta c_{r}: \quad$ Uncertainty of $c_{r}$

$c_{m}$ : Unit recycling cost from customers to the manufacturer

$\Delta c_{m}: \quad$ Uncertainty of $c_{m}$

$c_{t}$ : $\quad$ Unit recycling cost from customers to 3PRP

$\Delta c_{t}: \quad$ Uncertainty of $c_{t}$

$c_{m t}$ : Unit recycling cost from 3PRP to the

manufacturer

$\Delta c_{m t}: \quad$ Uncertainty of $c_{m t}$

$c_{q}: \quad$ Unit return cost

$\Delta c_{q}: \quad$ Uncertainty of $c_{q}$

$c_{d}: \quad$ Unit disposal cost

$\Delta c_{d}: \quad$ Uncertainty of $c_{d}$

$c_{s}: \quad$ Retailer's unit ordering cost

$\Delta c_{s}: \quad$ Uncertainty of $c_{s}$

$R_{i}$ : $\quad$ The $i$ th fuzzy rule, $i=1,2, \ldots, r$

$r$ : The number of fuzzy rules

$M_{j}^{i}$ : $\quad$ The fuzzy set, $j=1,2, \ldots, n$

$\tau_{e}: \quad \quad$ The $e$ th lead time, $e=1,2, \ldots, g$

$g: \quad$ The number of lead times

$n$ : $\quad$ The number of node enterprises

$M_{j}^{i}\left(x_{j}(k)\right)$ : The grade of membership of $x_{j}(k)$ in the fuzzy set $M_{j}^{i}$

$\mu_{i}(\mathbf{x}(k)): \quad$ The membership degree of the $i$ th rule

$\|\cdot\|_{2}: \quad \ell_{2}[0, \infty)$ norm

$\mathbf{G}_{c}: \quad$ The $c$ th MORG, $c=1,2, \ldots, \prod_{j=1}^{n}\left(m_{j}-1\right)$

$I_{c}: \quad$ The set of the rule numbers in $\mathbf{G}_{c}$

$m_{j}$ : The number of the fuzzy partitions of the $j$ th input variable

$F_{1}^{t}\left(x_{1}(k)\right):$ The fuzzy partitions of $x_{1}(k), t=1,2$

$F_{2}^{s}\left(x_{2}(k)\right)$ : The fuzzy partitions of $x_{2}(k), s=1,2$

$D_{0 m}$ : Television manufacturer's safe inventory

$D_{1 m}$ : Television manufacturer's expected inventory

$D_{m \text { max }}$ : Television manufacturer's maximum inventory

$D_{0 r}: \quad$ Television retailer's safe inventory

$D_{1 r}$ : $\quad$ Television retailer's expected inventory

$D_{r \text { max }}$ : Television retailer's maximum inventory.

\section{Data Availability}

The simulation data used to support the findings of this study are included within the manuscript. These data are restricted by the China Video Industry Association in order to protect trade secrets. Data can be available from http:// www.cvianet.org.cn for researchers who meet the criteria for access to confidential data.

\section{Conflicts of Interest}

The authors declare that they have no conflicts of interest.

\section{Acknowledgments}

The authors are grateful for supports from the Shandong Provincial Social Science Plan Foundation, China (no. 19BYSJ13) and the Shandong Provincial Natural Science Foundation, China (no. ZR2018 MG004).

\section{References}

[1] D. Yang and T. Xiao, "Pricing and green level decisions of a green supply chain with governmental interventions under fuzzy uncertainties," Journal of Cleaner Production, vol. 149, pp. 1174-1187, 2017.

[2] N. M. Modak and P. Kelle, "Using social work donation as a tool of corporate social responsibility in a closed-loop supply chain considering carbon emissions tax and demand uncertainty," Journal of the Operational Research Society, pp. 1-17, 2019.

[3] M. Zhalechian, R. Tavakkoli-Moghaddam, B. Zahiri, and M. Mohammadi, "Sustainable design of a closed-loop location-routing-inventory supply chain network under mixed uncertainty," Transportation Research Part E: Logistics and Transportation Review, vol. 89, pp. 182-214, 2016.

[4] X.-X. Zheng, Z. Liu, K. W. Li, J. Huang, and J. Chen, "Cooperative game approaches to coordinating a three-echelon closed-loop supply chain with fairness concerns," International Journal of Production Economics, vol. 212, pp. 92-110, 2019.

[5] I. H. Hong and J. S. Yeh, "Modeling closed-loop supply chains in the electronics industry: a retailer collection application," Transportation Research Part E: Logistics and Transportation Review, vol. 48, no. 4, pp. 817-829, 2012.

[6] J. Wei and J. Zhao, "Reverse channel decisions for a fuzzy closed-loop supply chain," Applied Mathematical Modelling, vol. 37, no. 3, pp. 1502-1513, 2013.

[7] A. Allah, M. S. Moshtagh, and I. Moon, "Pricing, product quality, and collection optimization in a decentralized closedloop supply chain with different channel structures: game theoretical approach," Journal of Cleaner Production, vol. 189, pp. $406-431,2018$.

[8] L. Shi and X. Ma, "Strategy analysis of closed loop supply chain for scrapped medical equipment based on hybrid recycling model," Advanced Materials Research, vol. 219-220, pp. 722-726, 2011.

[9] X. Ma and R. L. Liu, "Notice of retraction game model of closed loop supply chain under hybrid recycling strategy based on remanufacturing," in Proceedings of the IEEE International Conference on Computer Science and Information Technology, vol. 4, pp. 78-81, Chengdu, China, July 2010.

[10] N. A. D. Do, P. Nielsen, Z. Michna, and I. E. Nielsen, "Quantifying the bullwhip effect of multi-echelon system with stochastic dependent lead time," in Advances in Production Management Systems, B. Grabot, B. Vallespir, S. Gomes, A. Bouras, and D. Kiritsis, Eds., vol. 438, pp. 419-426, Springer, Berlin, Heidelberg, 2014. 
[11] S. Agrawal, R. N. Sengupta, and K. Shanker, "Impact of information sharing and lead time on bullwhip effect and onhand inventory," European Journal of Operational Research, vol. 192, no. 2, pp. 576-593, 2009.

[12] J. G. Kim, D. Chatfield, T. P. Harrison, and J. C. Hayya, "Quantifying the bullwhip effect in a supply chain with stochastic lead time," European Journal of Operational Research, vol. 173, no. 2, pp. 617-636, 2006.

[13] N. M. Modak and P. Kelle, "Managing a dual-channel supply chain under price and delivery-time dependent stochastic demand," European Journal of Operational Research, vol. 272, no. 1, pp. 147-161, 2019.

[14] C. Li and S. Liu, "A robust optimization approach to reduce the bullwhip effect of supply chains with vendor order placement lead time delays in an uncertain environment," Applied Mathematical Modelling, vol. 37, no. 3, pp. 707-718, 2013.

[15] D. Fu, C. Ionescu, E.-H. Aghezzaf, and R. De Keyser, "Quantifying and mitigating the bullwhip effect in a benchmark supply chain system by an extended prediction selfadaptive control ordering policy," Computers \& Industrial Engineering, vol. 81, pp. 46-57, 2015.

[16] C. A. G. Salcedo, A. I. Hernandez, R. Vilanova, and J. H. Cuartas, "Inventory control of supply chains: mitigating the bullwhip effect by centralized and decentralized internal model control approaches," European Journal of Operational Research, vol. 224, no. 2, pp. 261-272, 2013.

[17] C. E. Riddalls and S. Bennett, "Production-inventory system controller design and supply chain dynamics," International Journal of Systems Science, vol. 33, no. 3, pp. 181-195, 2002.

[18] X.-Y. Huang, N.-N. Yan, and R.-Z. Qiu, "Dynamic models of closed-loop supply chain and robust $H_{\infty}$ control strategies," International Journal of Production Research, vol. 47, no. 9, pp. 2279-2300, 2009.

[19] S. T. Zhang, X. Li, and C. Y. Zhang, "A fuzzy control model for restraint of bullwhip effect in uncertain closed-loop supply chain with hybrid recycling channels," IEEE Transactions on Fuzzy Systems, vol. 25, no. 2, pp. 475-482, 2017.

[20] S. F. Alamdar, M. Rabbani, and J. Heydari, "Pricing, collection, and effort decisions with coordination contracts in a fuzzy, three-level closed-loop supply chain," Expert Systems with Applications, vol. 104, pp. 261-276, 2018.

[21] T. Takagi and M. Sugeno, "Fuzzy identification of systems and its applications to modeling and control," IEEE Transactions on Systems, Man, and Cybernetics, vol. SMC-15, no. 1, pp. 116-132, 1985.

[22] Z.-H. Xiu and G. Ren, "Stability analysis and systematic design of Takagi-Sugeno fuzzy control systems," Fuzzy Sets and Systems, vol. 151, no. 1, pp. 119-138, 2005.

[23] X. D. Liu and Q. L. Zhang, "Approaches to quadratic stability conditions and $H_{\infty}$ control designs for T-S fuzzy systens," IEEE Transactions on Fuzzy Systems, vol. 11, no. 6, pp. 830839, 2003.

[24] S. T. Zhang, Y. T. Hou, S. Q. Zhang, and M. Zhang, "Fuzzy control model and simulation for nonlinear supply chain system with lead times," Complexity, vol. 2017, p. 11, 2017.

[25] Z. Liu, K. W. Li, B.-Y. Li, J. Huang, and J. Tang, "Impact of product-design strategies on the operations of a closed-loop supply chain," Transportation Research Part E: Logistics and Transportation Review, vol. 124, pp. 75-91, 2019. 


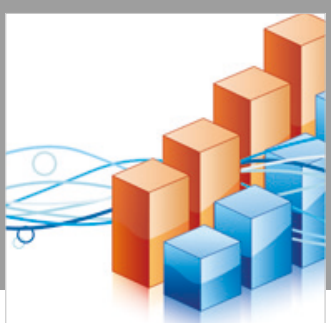

Advances in

Operations Research

\section{-n-m}
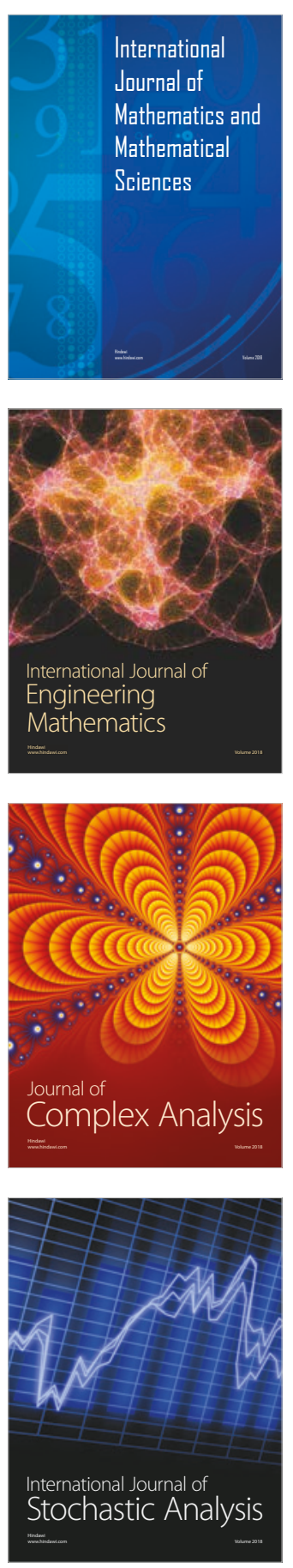
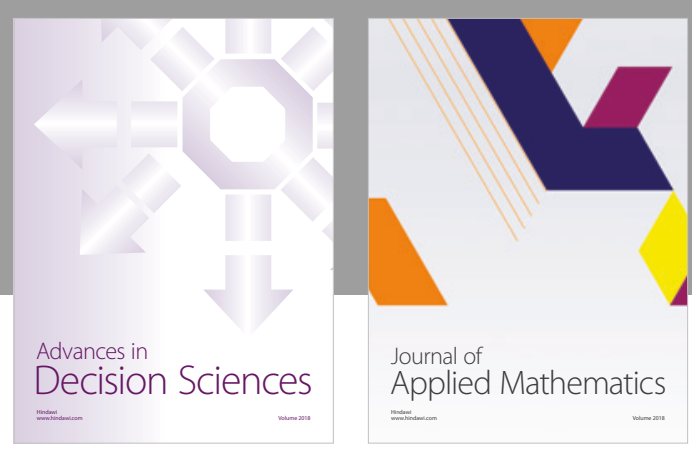

Journal of

Applied Mathematics
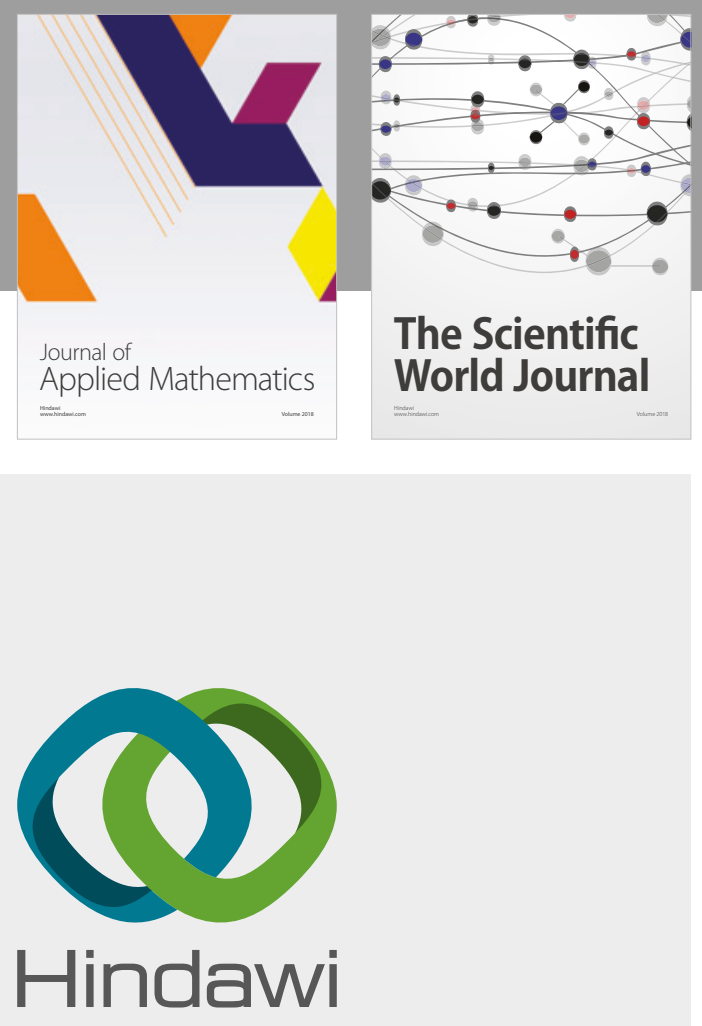

Submit your manuscripts at

www.hindawi.com

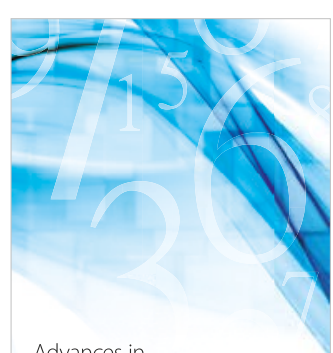

Advances in
Numerical Analysis
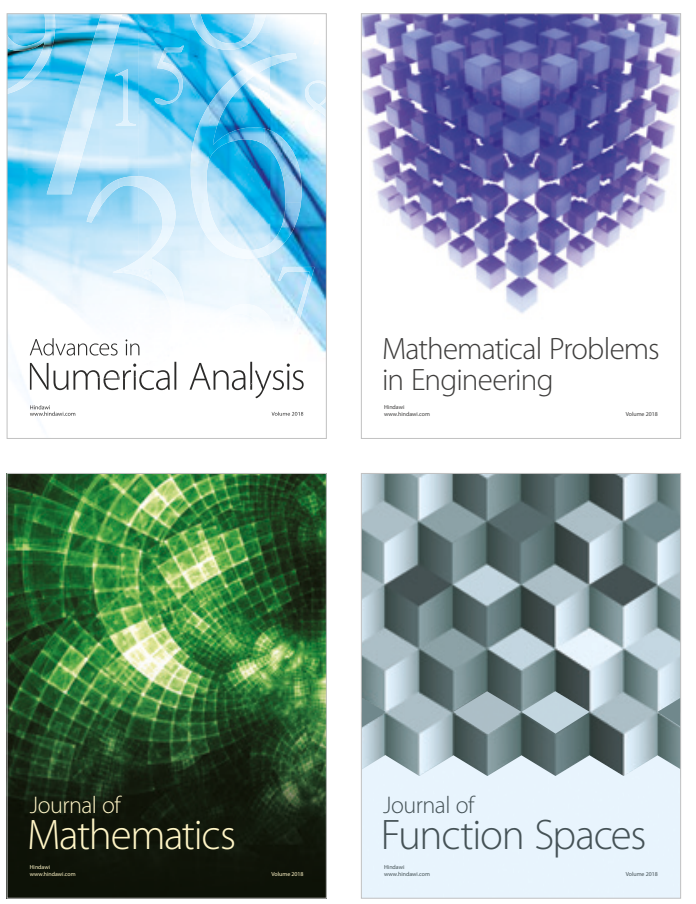

Mathematical Problems in Engineering

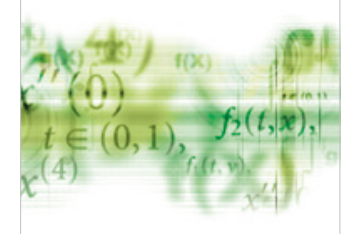

International Journal of

Differential Equations

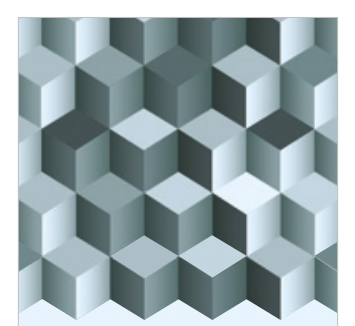

Journal of

Function Spaces
The Scientific

World Journal

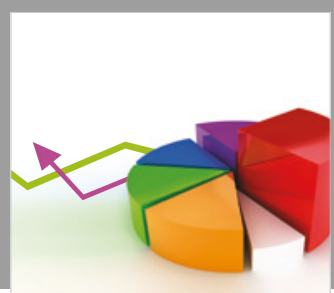

Journal of

Probability and Statistics
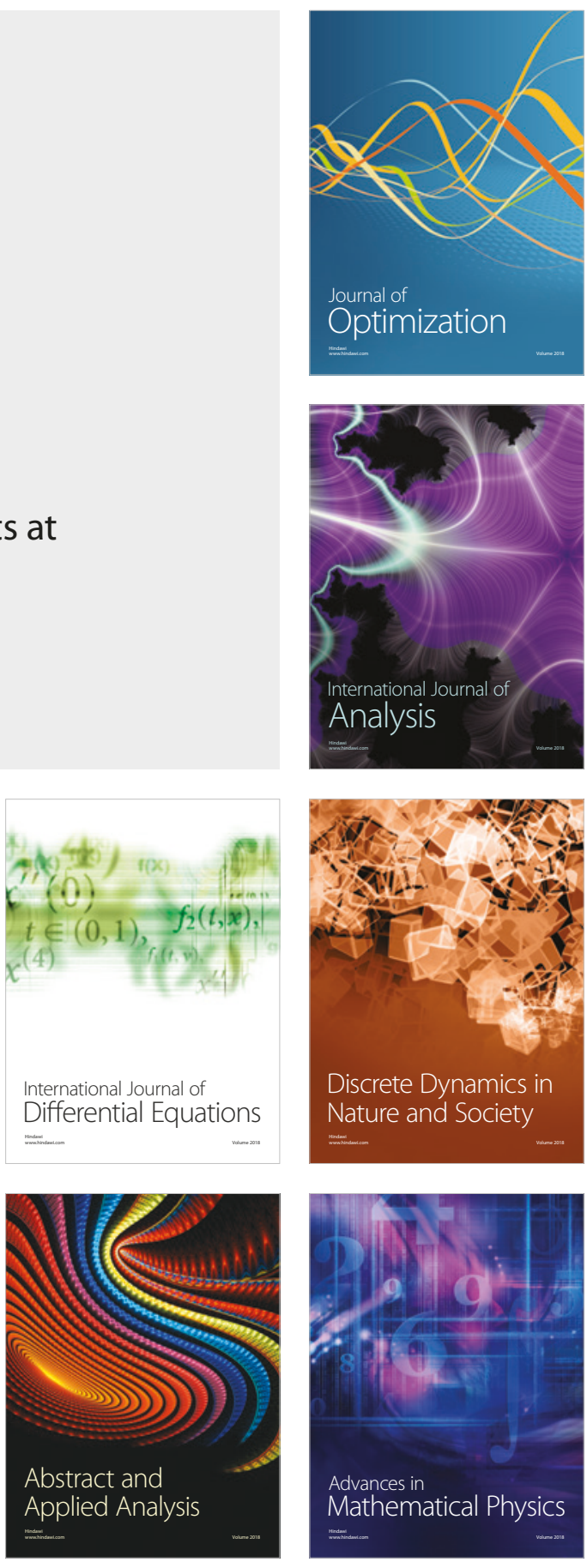